\title{
BLOCH WAVE HOMOGENIZATION OF LINEAR ELASTICITY SYSTEM
}

\author{
Sista Sivaji Ganesh ${ }^{1, *}$ And Muthusamy Vanninathan ${ }^{1}$
}

\begin{abstract}
In this article, the homogenization process of periodic structures is analyzed using Bloch waves in the case of system of linear elasticity in three dimensions. The Bloch wave method for homogenization relies on the regularity of the lower Bloch spectrum. For the three dimensional linear elasticity system, the first eigenvalue is degenerate of multiplicity three and hence existence of such a regular Bloch spectrum is not guaranteed. The aim here is to develop all necessary spectral tools to overcome these difficulties. The existence of a directionally regular Bloch spectrum is proved and is used in the homogenization. As a consequence an interesting relation between homogenization process and wave propagation in the homogenized medium is obtained. Existence of a spectral gap for the directionally regular Bloch spectrum is established and as a consequence it is proved that higher modes apart from the first three do not contribute to the homogenization process.
\end{abstract}

Mathematics Subject Classification. 35B27, 73B27, 74B05.

Received July 12, 2004. Revised December 23, 2004.

\section{INTRODUCTION}

In this article, we analyze the homogenization process of periodic structures using Bloch waves in the case of linear elasticity system in three dimensions. As is well known, homogenization process is concerned with macroscopic approximations of heterogeneous media. We refer the reader to the books $[4,11,14,23]$ for a beautiful analysis of this subject. To carry out the homogenization process various methods have been introduced in the literature. They include the methods of multiscale asymptotic expansions [4], oscillating test functions [16], two-scale convergence $[1,17], \Gamma$-convergence [11].

In contrast to the above physical space methods, Conca and Vanninathan, in their paper [8], have followed a purely Fourier approach using Bloch waves in the case of scalar selfadjoint problem. Their analysis has been extended to the non-selfadjoint case in [25]. For applications of the Bloch wave method, we cite a few references $[2,3,7-9,24]$. This method has also given rise to one fundamental object called Bloch approximation in the context of both theoretical and numerical aspects of homogenization $[5,6]$. In the literature, one also sees some phase space methods to homogenization: H-measures [26], defect measures [12], Wigner measures [13].

In [8], the authors work with the usual ordered Bloch spectrum and they prove the regularity of the first eigenvalue and eigenmode for small momenta $|\eta|$ and then use it to prove the required homogenization result.

\footnotetext{
Keywords and phrases. Bloch waves, homogenization, linear elasticity.

1 IISc-TIFR Mathematics Programme, TIFR Centre, P.O. Box 1234, Bangalore 560012, India; siva@math.tifrbng.res.in; vanni@math.tifrbng.res.in

* Thanks Council for Scientific and Industrial Research (CSIR), New Delhi and a part of this work was done when SSG was a CSIR fellow.
} 
In fact, the homogenized matrix is a multiple of the Hessien of the first eigenvalue at $\eta=0$. In the case of elasticity system, we have possible presence of transverse and longitudinal waves which did not exist in the scalar case of [8]. This gives rise to new difficulties in applying the Bloch wave method. Mathematically, the main difficulty stems from the multiplicity of the lowest eigenvalue. It is three in the present case. Another hurdle is that we have the presence of a vectorial perturbation parameter $\eta$. In such a case, regularity results are not guaranteed and the whole method seems to break down even at a heuristic level simply because the expression of the homogenized matrix does not make sense anymore. This non-regularity is not just a mathematical artifact but has some physical significance [10]. We examine this issue in the case of isotropic homogeneous medium in Section 2. It is proved that there is no smooth choice of eigenvalue-eigenvector pair near $\eta=0$ whether we demand ordering of eigenvalues or not. In fact, we obtain a necessary condition for a smooth choice to exist and it is easily seen that it is not satisfied. The upshot of the above analysis is the observation of directional regularity of the spectrum. We conclude thus that the appropriate regularity to be sought in the case of systems is that of directional regularity. This also enables us to overcome at the same time the difficulty posed by vectorial perturbation parameter. The goal of the paper is to exhibit such a spectrum and exploit it in the homogenization process. We mention that Turbé [27] applied Bloch decomposition to homogenize periodic elastic media. Compared to [27], this paper deals with different mathematical aspects of the problem and at the same time, elaborates certain results of [27].

\section{The plan of the paper}

In the Section 1, we introduce the shifted operator $A(\eta)$, for $\eta \in \mathbb{R}^{3}$, associated with the linear elasticity system. We then prove the existence of ordered Bloch spectrum (Lem. 1.1) and discuss the lack of regularity. Then fixing an arbitrary direction $\hat{\eta} \in \mathbb{R}^{3}$, we prove in Theorem 1.6 the existence of eigenvalues and eigenvectors of $A(\rho \hat{\eta})$ depending analytically on the scalar variable $\rho$. This is the directional regularity that we are seeking. We call them Rellich branches. It is clear that this indexing of the spectrum need not respect the usual ordering of eigenvalues.

The Section 2 is devoted to obtaining Bloch decomposition of the space $\mathbf{H}^{-1}\left(\mathbb{R}^{3}\right)$ as this is required in the homogenization process. Such a decomposition for $\mathbf{L}^{2}\left(\mathbb{R}^{3}\right)$ is more classical [4]. From this, we deduce an appropriate strengthened version of the decomposition for $\mathbf{H}^{1}\left(\mathbb{R}^{3}\right)$ and then we treat the space $\mathbf{H}^{-1}\left(\mathbb{R}^{3}\right)$ by duality (Th. 2.4).

In Section 3, we compute (Lem. 3.1) the Hessien of the eigenvalue in each direction. These individual Hessiens nicely patch up to define the homogenized matrix via what we call propagation condition. It has an interesting connection with the propagation of monochromatic waves in the homogenized medium (Lem. 3.3). Such features were not clear in the scalar case of [8].

Section 4 contains just one result which analyzes the asymptotic behaviour of the first three Bloch modes. The analogous property in the scalar case simply states that the first Bloch transform tends to Fourier transform [8]. As can be seen from Lemma 4.1, the case of systems is more complicated.

In Section 5, we state and prove the homogenization result (Th. 5.1) as a by-product of our foregoing analysis.

In Section 6, we establish spectral gap between the first three Bloch modes and the rest for the directionally regular Rellich spectrum (Lem. 6.3) and then use it to show that higher Bloch modes, apart from the first three, do not contribute to homogenization limit (Th. 6.1).

\section{THE SHIFTED OPERATOR AND ITS SPECTRUM}

The periodically varying medium is represented by the operator $A$ formally acting on vector functions $\boldsymbol{\Psi}(y)=$ $\left(\psi_{1}(y), \psi_{2}(y), \psi_{3}(y)\right)$, given by

$$
(A \boldsymbol{\Psi})_{p} \equiv-\frac{\partial}{\partial y_{q}}\left[\mathfrak{a}_{p q r s}(y) \mathfrak{e}_{r s}(\boldsymbol{\Psi}(y))\right] \equiv-\frac{\partial}{\partial y_{q}}\left[\mathfrak{a}_{p q r s}(y) \frac{\partial \psi_{r}}{\partial y_{s}}(y)\right], \quad p=1,2,3
$$


where strain tensor $\mathfrak{e}_{r s}(\boldsymbol{\Psi})$ is given by

$$
\mathfrak{e}_{r s}(\boldsymbol{\Psi})=\frac{1}{2}\left(\frac{\partial \psi_{r}}{\partial y_{s}}+\frac{\partial \psi_{s}}{\partial y_{r}}\right), r, s=1,2,3
$$

and the components of the elastic tensor $\mathfrak{a}_{p q r s}, p, q, r, s \in\{1,2,3\}$, are real valued and $Y$-periodic $\left(Y=\left[0,2 \pi\left[^{3}\right)\right.\right.$ functions such that:

$$
\left.\begin{array}{c}
\mathfrak{a}_{q p r s}=\mathfrak{a}_{p q r s}=\mathfrak{a}_{r s p q}=\mathfrak{a}_{p q s r}, \\
\mathfrak{a}_{p q r s} \in L_{\#}^{\infty}(Y),\left|\mathfrak{a}_{p q r s}(y)\right| \leq \alpha^{\prime} \text { a.e. } y \in Y, \text { and } \\
\exists \alpha>0: \mathfrak{a}_{p q r s}(y) \varepsilon_{p q} \bar{\varepsilon}_{r s} \geq \alpha \varepsilon_{p q} \bar{\varepsilon}_{p q} \text { a.e. } y \in Y, \\
\text { or every complex-valued }(3 \times 3) \text { symmetric matrix } \varepsilon,
\end{array}\right\}
$$

where we followed the usual summation convention over repeated indices and we recall that a function $u$ defined on $\mathbb{R}^{3}$ is said to be $Y$-periodic if

$$
u\left(x+2 \pi \mathbf{e}_{j}\right)=u(x) \text { a.e. } x \in \mathbb{R}^{3}, \forall j=1,2,3,
$$

where $\mathbf{e}_{j}, j=1,2,3$ denotes the standard ordered basis for $\mathbb{R}^{3}$. Throughout this article, the subscript \# for a function space indicates that the space consists of periodic functions.

Bloch waves are defined to be eigenfunctions $\boldsymbol{\Psi}(\eta)$ of the operator $A$ indexed by a parameter $\eta \in \mathbb{R}^{3}$, satisfying the $(\eta, Y)$-periodic condition, i.e.,

$$
\boldsymbol{\Psi}(y+2 \pi p ; \eta)=\mathrm{e}^{2 \pi i p \cdot \eta} \boldsymbol{\Psi}(y ; \eta) \quad \forall p \in \mathbb{Z}^{3}, y \in \mathbb{R}^{3}
$$

Since the $(\eta, Y)$-periodic condition in (1.5) is invariant under translations by elements of $\mathbb{Z}^{3}$ in the $\eta$-variable, $\eta$ may be restricted to the set $Y^{\prime}=[-1 / 2,1 / 2)^{3}$ which is referred to as the dual cell.

Introducing Floquet ansatz $\boldsymbol{\Psi}(y ; \eta)=\mathrm{e}^{i y \cdot \eta} \boldsymbol{\Phi}(y ; \eta)$ where $\boldsymbol{\Phi}(, ; \eta)$ is $Y$-periodic, we have the following eigenvalue problem satisfied by $\boldsymbol{\Phi}(, ; \eta)$ :

$$
\left.\begin{array}{c}
A(\eta) \boldsymbol{\Phi}(. ; \eta)=\lambda(\eta) \boldsymbol{\Phi}(. ; \eta) \quad \text { in } \mathbb{R}^{3}, \\
\text { and } \boldsymbol{\Phi}(. ; \eta) \text { is Y-periodic, }
\end{array}\right\}
$$

where the operator $A(\eta)$ is the so-called shifted operator and is given by

$$
A(\eta)(\mathbf{u}) \stackrel{\text { def }}{=}-\left(\frac{\partial}{\partial y_{q}}+i \eta_{q}\right)\left\{\mathfrak{a}_{p q r s}(y) \frac{1}{2}\left[\left(\frac{\partial}{\partial y_{s}}+i \eta_{s}\right) u_{r}+\left(\frac{\partial}{\partial y_{r}}+i \eta_{r}\right) u_{s}\right]\right\}
$$

For the rest of this section, we are interested in the spectrum of $A(\eta)$ considered as an unbounded operator in $\mathbf{L}_{\#}^{2}(Y)$ and its dependence on $\eta \in Y^{\prime}$.

\subsection{Existence of ordered spectrum of $A(\eta)$ and its lack of regularity}

We start with a comment on the title of this subsection. The word "ordered spectrum" is used to remind us that we will be discussing about the spectrum of $A(\eta)$ wherein the eigenvalues are non-decreasing with the increasing index. It is well-known that $A(\eta)$ is a selfadjoint operator in $\mathbf{L}_{\#}^{2}(Y)$ and is non-negative definite (see [20]) (a consequence of symmetry and ellipticity assumptions on the elastic tensor given in (1.4)). As a consequence of the compact embedding of $\mathbf{H}_{\#}^{1}(Y)$ into $\mathbf{L}_{\#}^{2}(Y),\left(A(\eta)+C_{*} I\right)^{-1}$ is a compact operator on $\mathbf{L}_{\#}^{2}(Y)$ 
for some $C_{*}>0$. All these properties are sufficient to apply spectral theorem for selfadjoint operators with compact resolvent and we have the following result asserting the ordered spectrum (see [20]):

Lemma 1.1 (existence of ordered spectrum). For $\eta \in Y^{\prime}$, there exist sequences of eigenvalues $\left\{\mu_{k}(\eta)\right\}_{k=1}^{\infty}$ and eigenvectors $\left\{\boldsymbol{\Theta}_{k}(\eta)\right\}_{k=1}^{\infty}$ belonging to $\mathbf{H}_{\#}^{1}(Y)$ for the problem (1.6) satisfying $0 \leq \mu_{1}(\eta) \leq \mu_{2}(\eta) \leq \ldots \leq$ $\mu_{k}(\eta) \leq \ldots \rightarrow \infty$, with each eigenvalue being repeated according as its multiplicity, and $\left\{\boldsymbol{\Theta}_{k}(\eta)\right\}_{k=1}^{\infty}$ forms an orthonormal basis in $\mathbf{L}_{\#}^{2}(Y)$.

\section{Lack of regularity of the ordered spectrum}

For fixed $k \in \mathbb{N}$, the eigenvalues $\mu_{k}(\eta)$, owing to their variational characterization (see [8]) via Courant-Fischer minimax principle, are Lipschitz continuous functions of $\eta \in Y^{\prime}$. This global regularity is not enough in the process of homogenization (see [8]); we need higher local regularity. Of course, we should mention that only the regularity of the first eigenvalue near $\eta=0$ was needed in the case of scalar selfadjoint operator case of [8]. This was the only eigenbranch emanating from the least eigenvalue of $A(0)$ which is equal to zero. In the present case, the least eigenvalue which is again equal to zero has multiplicity three. Therefore, we need to study the splitting of this multiple eigenvalue i.e., analysis of spectrum of perturbation near a multiple eigenvalue.

The perturbation theory for linear operators is very classical and it is known that the spectrum need not reflect the regularity of the operator in the perturbation variable; more so, if the perturbation parameter is not a scalar and if the eigenvalue under study is not simple and is illustrated by the following classical example due to Rellich (for example, see $[15,19]$ ).

Example 1.2. Let us consider the family of diagonalizable matrices depending on a parameter $\eta \in \mathbb{R}^{2}, \eta=$ $\left(\eta_{1}, \eta_{2}\right) \in \mathbb{R}^{2} \longmapsto\left(\begin{array}{cc}\eta_{1} & \eta_{2} \\ \eta_{2} & -\eta_{1}\end{array}\right) \in M_{2}(\mathbb{R})$. We note that this is a family of symmetric matrices, and hence are diagonalizable. Further, the matrix entries depend linearly on $\eta$. It is easy to see that eigenvalues are given by $\mu_{1}(\eta)=-\sqrt{\eta_{1}^{2}+\eta_{2}^{2}}$ and $\mu_{2}(\eta)=\sqrt{\eta_{1}^{2}+\eta_{2}^{2}}$. Note that when the parameter $\eta$ is equal to 0 , the two eigenvalues coincide and the common value is 0 with eigenspace $\mathbb{R}^{2}$, whereas for $\eta \neq 0, \mu_{1}(\eta)<\mu_{2}(\eta)$. It is obvious that they are Lipschitz continuous functions of the parameter $\eta$ but not differentiable at $\eta=0$. It can be easily seen that eigenvectors can not be chosen to be continuous with respect to $\eta$ at $\eta=0$.

Next, we present a particular case of linear elasticity system wherein we see clearly the lack of regularity.

Example 1.3 (homogeneous isotropic medium). The coefficients $\mathfrak{a}_{\text {pqrs }}(y)$ representing the isotropic homogeneous medium are constants, given by

$$
\mathfrak{a}_{p q r s}(y) \equiv \lambda \delta_{p q} \delta_{r s}+\mu\left(\delta_{p r} \delta_{q s}+\delta_{p s} \delta_{q r}\right), \forall y \in Y,
$$

where the Lamé coefficients $\lambda, \mu$ have the usual properties: $\mu>0$ and $3 \lambda+2 \mu>0$.

Regarding the ordered spectrum in this case, Lemma 1.1 asserts the existence of eigenvalues and eigenvectors of the operator $A(\eta)$ defined by (1.7). Since eigenvectors are $Y$-periodic, we expand them in Fourier series, and substitute in the eigenvalue-eigenvector relation for the operator $A(\eta)$ defined by $(1.7)$, and we reduce the operator problem to the spectral problem of the following numerical matrix equation.

$$
(\lambda+\mu)(\mathbf{m}+\eta) \otimes(\mathbf{m}+\eta)+\mu\|\mathbf{m}+\eta\|^{2} I_{3 \times 3}, \text { where } \mathbf{m} \in \mathbb{Z}^{3}
$$

where $(\mathbf{m}+\eta) \otimes(\mathbf{m}+\eta)$ denotes a matrix whose $(i, j)$-th entry is $\left(m_{i}+\eta_{i}\right)\left(m_{j}+\eta_{j}\right)$, and $I_{3 \times 3}$ denotes the $3 \times 3$ identity matrix. Even though $\mathbf{m}$ is repeated in (1.9), there is no summation with respect to $\mathbf{m}$. The eigenelements of the operator (1.7) in the case of (1.8) are as follows:

For $\mathbf{m} \in \mathbb{Z}^{3}, \eta \in Y^{\prime}$ such that $(\mathbf{m}, \eta) \neq(0,0)$,

(i) (transverse waves) $\mu\|\mathbf{m}+\eta\|^{2}$ is an eigenvalue of multiplicity two, with eigenvectors $\frac{\mathbf{L}}{(2 \pi)^{3 / 2}} \mathrm{e}^{i \mathbf{m} \cdot y}$, where $\mathbf{L} \in \mathbb{R}^{3}$ is a unit vector satisfying $\mathbf{L} .(\mathbf{m}+\eta)=0$; 
(ii) (longitudinal waves) $(\lambda+2 \mu)\|\mathbf{m}+\eta\|^{2}$ is an eigenvalue with eigenvector $\frac{\mathbf{K}}{(2 \pi)^{3 / 2}} \mathrm{e}^{i \mathbf{m} \cdot y}$, where $\mathbf{K} \in \mathbb{R}^{3}$ is a unit vector parallel to $\mathbf{m}+\eta$,

and for $(\mathbf{m}, \eta)=(0,0)$, zero is an eigenvalue with eigenspace $\mathbb{C}^{3}$.

Moreover, $\forall \eta \in Y^{\prime}$ these eigenvectors form a complete orthonormal basis for $\mathbf{L}_{\#}^{2}(Y)$ as $\mathbf{m} \in \mathbb{Z}^{3}$ varies.

In the general case of media defined by (1.4) with symmetries (1.3), when the parameter $\eta$ is equal to 0 , any non-zero $\mathbf{u} \equiv$ constant vector in $\mathbb{C}^{3}$ is an eigenvector of the operator $A(0)$, corresponding to the eigenvalue 0 and hence the eigenspace is of dimension three. For each $k=1,2,3$, let $\mu_{k}(\eta)$ and $\boldsymbol{\Theta}_{k}(\eta)$ be the first three of the ordered eigenvalues and corresponding eigenvectors of the shifted operator (1.7), whose existence is assured by Lemma 1.1. Then $\mu_{1}(0)=\mu_{2}(0)=\mu_{3}(0)=0$, and $\boldsymbol{\Theta}_{1}(0), \boldsymbol{\Theta}_{2}(0), \boldsymbol{\Theta}_{3}(0)$ are such that they form an orthogonal basis for $\mathbb{C}^{3}$.

In the special case of homogeneous isotropic media defined by (1.8), we can identify the first three ordered eigenvalues $\mu_{1}(\eta), \mu_{2}(\eta), \mu_{3}(\eta)$ for $\eta$ in a sufficiently small neighbourhood of zero. In fact, in the above computations, the eigenvalue can be zero if and only if $\mathbf{m}+\eta=0$, i.e., $\mathbf{m}=-\eta \in Y^{\prime}$, therefore $\mathbf{m}=0$ and $\eta=0$. Thus, we get, for $\|\eta\|$ small, $\mu_{1}(\eta)=\mu_{2}(\eta)=\mu\|\eta\|^{2}$, and $\mu_{3}(\eta)=(\lambda+2 \mu)\|\eta\|^{2}$ (since $\lambda+2 \mu=$ $\left(\lambda+\frac{2}{3} \mu\right)+\frac{1}{3} \mu+\mu>\mu$ by our assumptions on Lamé coefficients).

For $k=1,2,3$, we claim that there cannot be a continuous choice of $\boldsymbol{\Theta}_{k}(\eta)$, unit eigenvectors corresponding to the eigenvalue $\mu_{k}(\eta)$ for $\eta$ near zero. The claim will be proved only for the case $k=3$ since the arguments are more transparent. The claim will follow for the cases $k=1,2$ in a similar fashion.

Indeed, there is a unique choice for longitudinal wave $\boldsymbol{\Theta}_{3}(\eta)$ (up to sign) when $\eta \neq 0$, as $\mu_{3}(\eta)$ is simple, given by (see (ii) above)

$$
\boldsymbol{\Theta}_{3}(\eta)=\frac{1}{(2 \pi)^{3 / 2} \sqrt{\eta_{1}^{2}+\eta_{2}^{2}+\eta_{3}^{2}}}\left(\begin{array}{l}
\eta_{1} \\
\eta_{2} \\
\eta_{3}
\end{array}\right)
$$

Let us fix a unit vector $\hat{\eta} \in \mathbb{R}^{3}$. For $\eta=\delta \hat{\eta}$ with $\delta>0$, the above eigenvectors take the following form in terms of $\hat{\eta}$ :

$$
\boldsymbol{\Theta}_{3}^{+}(\eta)=\frac{1}{(2 \pi)^{3 / 2} \sqrt{\hat{\eta}_{1}^{2}+\hat{\eta}_{2}^{2}+\hat{\eta}_{3}^{2}}}\left(\begin{array}{c}
\hat{\eta}_{1} \\
\hat{\eta}_{2} \\
\hat{\eta}_{3}
\end{array}\right)
$$

which depend on $\hat{\eta}$ and not on $\delta$ (i.e., they are homogeneous of order zero) and hence is not a continuous function of $\eta$ at $\eta=0$.

However, a continuous (in fact, constant) choice of $\boldsymbol{\Theta}_{3}(\eta)$ can be made along each fixed direction $\hat{\eta} \in \mathbb{R}^{3}$, for example, $\boldsymbol{\Theta}_{3}^{+}(\eta)$ given above. This observation will be exploited later.

Even by foregoing the ordering of the eigenvalues, we only have eigenvalues which are continuous with respect to $\eta$ near $\eta=0$ and we cannot choose eigenvectors depending continuously on $\eta$ near $\eta=0$. Indeed, we observe that the only continuous eigenvalue branches near $\eta=0$ are given by $\mu\|\eta\|^{2}$ (of multiplicity two) and $(\lambda+2 \mu)\|\eta\|^{2}$ (simple eigenvalue) from the explicit computations made above. Now it is clear that there is no continuous choice of eigen vectors near $\eta=0$.

One may think that the above lack of regularity of eigenvector is due to the requirement that eigenvalues are arranged in increasing order. Actually, this is not so. In the following remark we make it precise.

Remark 1.4 (necessary conditions for the existence of smooth eigenelements near $\eta=0$ ). In the general case of media defined by (1.4), we remark that the corresponding homogenized medium should satisfy certain necessary conditions for the existence of smooth eigenvalue and eigenvector branches. More precisely, Let $\widetilde{\lambda_{k}}(\eta), k=1,2,3$ 
be eigenvalues with corresponding eigenvectors $\widetilde{\boldsymbol{\Theta}_{k}}(\eta), k=1,2,3$ satisfying all the following conditions:

$$
\left\{\begin{array}{l}
\lim _{\eta \rightarrow 0} \widetilde{\lambda}_{k}(\eta)=0, k=1,2,3, \\
\widetilde{\lambda_{k}}(\eta) \text { is } C^{2} \text { near } \eta=0, \widetilde{\boldsymbol{\Theta}_{k}}(\eta) \text { is } C^{2} \text { near } \eta=0, \\
\left\{\widetilde{\boldsymbol{\Theta}_{k}}(0): k=1,2,3\right\} \text { are eigenvectors of } A(0) \text { and form a basis of } \mathbb{C}^{3} .
\end{array}\right.
$$

Differentiating the eigenvalue equations (1.6), with respect to $\eta_{q}$ and $\eta_{s}$ and evaluating at $\eta=0$, we obtain the following relations:

$$
\frac{\partial^{2} \widetilde{\lambda_{k}}}{\partial \eta_{q} \partial \eta_{s}}(0) \widetilde{\boldsymbol{\Theta}_{k}}(y ; 0)=A_{q s} \widetilde{\boldsymbol{\Theta}_{k}}(y ; 0), k=1,2,3,
$$

where, the symmetric matrices $\left\{A_{q s}\right\}_{1 \leq q, s \leq 3}$ are given by

$$
A_{q s}=\left(\begin{array}{lll}
\mathfrak{b}_{1 q 1 s}+\mathfrak{b}_{1 s 1 q} & \mathfrak{b}_{1 q 2 s}+\mathfrak{b}_{1 s 2 q} & \mathfrak{b}_{1 q 3 s}+\mathfrak{b}_{1 s 3 q} \\
\mathfrak{b}_{2 q 1 s}+\mathfrak{b}_{2 s 1 q} & \mathfrak{b}_{2 q 2 s}+\mathfrak{b}_{2 s 2 q} & \mathfrak{b}_{2 q 3 s}+\mathfrak{b}_{2 s 3 q} \\
\mathfrak{b}_{3 q 1 s}+\mathfrak{b}_{3 s 1 q} & \mathfrak{b}_{3 q 2 s}+\mathfrak{b}_{3 s 2 q} & \mathfrak{b}_{3 q 3 s}+\mathfrak{b}_{3 s 3 q}
\end{array}\right)
$$

where the homogenized coefficients $\mathfrak{b}_{\text {pqrs }}$ are given by (3.1). We refer the reader to the section 3 for detailed computations. The relations (1.10) say that these matrices have common eigenvectors $\left\{\widetilde{\boldsymbol{\Theta}_{k}}(y ; 0): k=1,2,3\right\}$, which form a basis for $\mathbb{C}^{3}$ and hence the matrices must commute with each other. This is a necessary condition for the existence of smooth eigenvectors near $\eta=0$ as required above. In general, this condition is not satisfied. Let us consider the special case of isotropic homogeneous media defined by (1.8), the above matrices are

$$
\begin{gathered}
A_{11}=\left(\begin{array}{ccc}
\lambda+2 & \mu & 0 \\
0 & \mu & 0 \\
0 & 0 & \mu
\end{array}\right), A_{12}=\left(\begin{array}{ccc}
0 & \lambda+\mu & 0 \\
\lambda+\mu & 0 & 0 \\
0 & 0 & 0
\end{array}\right), A_{13}=\left(\begin{array}{ccc}
0 & 0 & \lambda+\mu \\
0 & 0 & 0 \\
\lambda+\mu & 0 & 0
\end{array}\right), \\
A_{23}=\left(\begin{array}{lll}
0 & 0 & 0 \\
0 & 0 & \lambda+\mu \\
0 & \lambda+\mu & 0
\end{array}\right), A_{22}=\left(\begin{array}{ccc}
\mu & 0 & 0 \\
0 & \lambda+2 \mu & 0 \\
0 & 0 & \mu
\end{array}\right), A_{33}=\left(\begin{array}{lll}
\mu & 0 & 0 \\
0 & \mu & 0 \\
0 & 0 & \lambda+2 \mu
\end{array}\right) .
\end{gathered}
$$

But, $A_{12}$ and $A_{23}$ commute with each other if and only if $\lambda+\mu=0$. Since $\lambda+\mu \neq 0$ by our assumptions on $\lambda$ and $\mu$, we conclude that a smooth choice of eigenvalues and eigenvectors is not possible in this case.

\subsection{Existence of eigenvalues and eigenvectors with directional regularity}

We investigate the possibility of choosing directionally regular eigenvectors in the more general case of operator $A(\eta)$ guided by the Example 1.3. In this paragraph, the existence of a directionally regular spectrum is proved (Th. 1.6). This notion of directionally regular spectrum is also consistent with the polarization phenomenon already observed in the case of isotropic homogeneous medium. This will be further explained in Section 3 .

In the sequel, it will be convenient to work with direction $\hat{\eta}$ defined below.

Let us note that for every non-zero $\eta \in Y^{\prime}$, there exists a unique $\rho \in \mathbb{R}$ and a unique vector $\hat{\eta}$ on the boundary of $Y^{\prime}$ whose first non-zero component is positive, such that $\eta=\rho \hat{\eta}$, and $|\rho|<1$. The set where $\hat{\eta}$ belongs will be denoted by $\partial Y_{+}^{\prime}$ and we have

$$
\partial Y_{+}^{\prime}=\left\{\hat{\eta} \in \partial Y^{\prime}: \hat{\eta}_{1}>0\right\} \cup\left\{\hat{\eta} \in \partial Y^{\prime}: \hat{\eta}_{1}=0, \hat{\eta}_{2}>0\right\} \cup\left\{\hat{\eta} \in \partial Y^{\prime}: \hat{\eta}_{1}=\hat{\eta}_{2}=0, \hat{\eta}_{3}>0\right\} .
$$

Geometrically $\hat{\eta}$ is the point where the straight line passing through the origin and $\eta$ meets $\partial Y_{+}^{\prime}$. Let $\eta \neq 0$ and $l$ be the least index such that $\eta_{l} \neq 0$. Then the $\rho, \eta$ and $\hat{\eta}$ are related by

$$
\eta=\rho \hat{\eta}, \quad \rho=2 \eta_{l}, \quad \hat{\eta}=\frac{1}{2 \eta_{l}} \eta
$$


We now fix a vector $\hat{\eta} \in \partial Y_{+}^{\prime}$. Now the operator $A(\eta)$ given by (1.7) is equal to $A(\rho \hat{\eta})$ which depends on a scalar parameter $\rho$. We first prove that a suitable extension $A(z \hat{\eta})$ of $A(\rho \hat{\eta})$ to $z \in \mathbb{C}$ is a holomorphic family of type (B) in the sense of Kato and then appeal to Kato-Rellich theorem to assert the existence of directionally regular spectrum. For the definition of a holomorphic family of sesquilinear forms, we refer to Kato's book [15]. We begin by recalling few notations. In this section, $(.)_{\mathbf{L}_{\#}^{2}(Y)}$ always denotes the scalar product in the Hilbert space $\mathbf{L}_{\#}^{2}(Y)$. The quadratic form $\mathbf{t}[\mathbf{u}]$ corresponding to a sesquilinear form $\mathbf{t}$ with domain $\mathfrak{D}(\mathbf{t}) \subseteq \mathbf{L}_{\#}^{2}(Y)$ is defined by

$$
\mathbf{t}[\mathbf{u}]:=\mathbf{t}[\mathbf{u}, \mathbf{u}] .
$$

The real and imaginary parts of $\mathbf{t}[\mathbf{u}]$ are denoted by $\Re \mathbf{t}[\mathbf{u}]$ and $\Im \mathbf{t}[\mathbf{u}]$ respectively. In our context, there exists a canonical family of sesquilinear forms associated with the operator $A(\rho \hat{\eta})$, namely, the family of sesquilinear forms $\mathbf{t}(z \hat{\eta})$ depending on $z \in \mathbb{C}$, with same domain given by $\mathfrak{D}(\mathbf{t}(z))=\mathbf{H}_{\#}^{1}(Y)$ contained in $\mathbf{L}_{\#}^{2}(Y)$, defined by

$$
\begin{aligned}
\mathbf{t}(z \hat{\eta})[\mathbf{u}, \mathbf{v}]= & \int_{Y} \mathfrak{a}_{p q r s}(y) \frac{\partial u_{q}}{\partial y_{p}} \frac{\partial \overline{v_{r}}}{\partial y_{s}} \mathrm{~d} y+i z \int_{Y} \mathfrak{a}_{p q r s}(y)\left(\hat{\eta}_{p} u_{q} \frac{\partial \overline{v_{r}}}{\partial y_{s}}-\hat{\eta}_{s} \overline{v_{r}} \frac{\partial u_{q}}{\partial y_{p}}\right) \mathrm{d} y \\
& +z^{2} \int_{Y} \mathfrak{a}_{p q r s}(y) \hat{\eta}_{p} \hat{\eta}_{s} u_{q} \overline{v_{r}} \mathrm{~d} y
\end{aligned}
$$

which when $z=\rho \in \mathbb{R}$, reduces to the sesquilinear form corresponding to the operator $A(\eta)$. In view of $|\rho|<1$, we restrict $z$ to the set $R:=\{z \in \mathbb{C}: z=a+i b,|a|<1,|b|<1\}$.

Theorem 1.5. $\mathbf{t}(z \hat{\eta})$ is a holomorphic family of type $(a)$.

Proof. The quadratic form corresponding to the sesquilinear form in (1.12) is

$$
\begin{aligned}
\mathbf{t}(z \hat{\eta})[\mathbf{u}]= & \int_{Y} \mathfrak{a}_{p q r s}(y) \frac{\partial u_{q}}{\partial y_{p}} \frac{\partial \overline{u_{r}}}{\partial y_{s}} \mathrm{~d} y+i z \int_{Y} \mathfrak{a}_{p q r s}(y)\left(\hat{\eta}_{p} u_{q} \frac{\partial \overline{u_{r}}}{\partial y_{s}}-\hat{\eta}_{s} \overline{u_{r}} \frac{\partial u_{q}}{\partial y_{p}}\right) \mathrm{d} y \\
& +z^{2} \int_{Y} \mathfrak{a}_{p q r s}(y) \hat{\eta}_{p} \hat{\eta}_{s} u_{q} \overline{u_{r}} \mathrm{~d} y
\end{aligned}
$$

\section{Step (i). $\mathbf{t}(z \hat{\eta})$ is sectorial}

The sectorial property of the form $\mathbf{t}(z \hat{\eta})$ is essentially a consequence of the ellipticity of the elastic tensor (see (1.4)). The generic constants $C^{*}, C_{*}$ etc. appearing in the estimates below are independent of $\mathbf{u}$ and $z \in R$. For some $C_{*}>\frac{\alpha}{4}$, the following inequality holds (see [20], for a proof):

$$
\Re \mathbf{t}(z \hat{\eta})[\mathbf{u}]+C_{*}\|\mathbf{u}\|_{\mathbf{L}_{\#}^{2}(Y)}^{2} \geq \frac{\alpha}{4}\|\mathbf{u}\|_{\mathbf{H}_{\#}^{1}(Y)}^{2} .
$$

We consider the new family of forms $\tilde{\mathbf{t}}(z \hat{\eta})$, with same domain as that of the family $\mathbf{t}(z \hat{\eta})$, namely $\mathbf{H}_{\#}^{1}(Y)$ contained in $\mathbf{L}_{\#}^{2}(Y)$, defined by

$$
\tilde{\mathbf{t}}(z \hat{\eta})[\mathbf{u}, \mathbf{v}]:=\mathbf{t}(z \hat{\eta})[\mathbf{u}, \mathbf{v}]+C_{*}(\mathbf{u}, \mathbf{v})_{\mathbf{L}_{\#}^{2}(Y)} .
$$

For the new family $\tilde{\mathbf{t}}(z \hat{\eta})$, the inequality (1.13) reads as

$$
\Re \tilde{\mathbf{t}}(z \hat{\eta})[\mathbf{u}] \geq \frac{\alpha}{4}\|\mathbf{u}\|_{\mathbf{H}_{\#}^{1}(Y)}^{2} .
$$


The following estimate holds for the imaginary part $\Im \tilde{\Im}(z \hat{\eta})[\mathbf{u}]$ of $\tilde{\mathbf{t}}(z \hat{\eta})[\mathbf{u}]$ for some $C^{*}>0$ :

$$
|\Im \tilde{\mathbf{t}}(z \hat{\eta})[\mathbf{u}]| \leq C^{*}\left(\Re \tilde{\mathbf{t}}(z \hat{\eta})[\mathbf{u}]-\frac{\alpha}{4}\|\mathbf{u}\|_{\mathbf{L}_{\#}^{2}(Y)}^{2}\right) .
$$

Thus, $\tilde{\mathbf{t}}(z \hat{\eta})$ is sectorial for each $z$ and uniformly with respect to $z \in R$. Since the addition of a scalar does not affect the sectorial nature, it follows that $\mathbf{t}(z \hat{\eta})$ is sectorial.

\section{Step (ii). $\mathbf{t}(z \hat{\eta})$ is closed}

It is an easy consequence of (1.14).

\section{Step (iii). $\mathbf{t}(z \hat{\eta})$ is a holomorphic family of type (a)}

We proved that each $\mathbf{t}(z \hat{\eta})$ is closed, sectorial and since $\mathbf{t}(z \hat{\eta})[\mathbf{u}]$ is a quadratic polynomial in $z$ for each fixed $\mathbf{u} \in \mathbf{H}_{\#}^{1}(Y)$, it follows that it is a holomorphic family of type (a).

Associated with each $\mathbf{t}(z \hat{\eta})$, there exists a unique m-sectorial operator with domain contained in $\mathbf{H}_{\#}^{1}(Y)$ thanks to the first representation theorem, a proof of which is available on page 322 of Kato's book [15]. The family of such operators associated with a holomorphic family of sesquilinear forms of type (a) is called a holomorphic family of type $(B)$. The aforementioned $m$-sectorial operator is given by

$$
A(z \hat{\eta})(\mathbf{u}) \equiv-\left(\frac{\partial}{\partial y_{q}}+i z \hat{\eta}_{q}\right)\left\{\mathfrak{a}_{p q r s}(y) \frac{1}{2}\left[\left(\frac{\partial}{\partial y_{s}}+i z \hat{\eta}_{s}\right) u_{r}+\left(\frac{\partial}{\partial y_{r}}+i z \hat{\eta}_{r}\right) u_{s}\right]\right\} .
$$

Since $\mathbf{t}(z \hat{\eta})^{*}=\mathbf{t}(\bar{z} \hat{\eta})$, where $\mathbf{t}(z \hat{\eta})^{*}$ is defined by $\mathbf{t}(z \hat{\eta})^{*}[\mathbf{u}, \mathbf{v}]:=\overline{\mathbf{t}(z \hat{\eta})[\mathbf{v}, \mathbf{u}]}$, we have $A(z \hat{\eta})^{*}=A(\bar{z} \hat{\eta})$. Thus the family $A(z \hat{\eta})$ is a selfadjoint holomorphic family of type $(B)$. Since the embedding of $\mathbf{H}_{\#}^{1}(Y)$ in $\mathbf{L}_{\#}^{2}(Y)$ is compact by Rellich's theorem, it follows that the operator $\left(A(z \hat{\eta})+C_{*} I\right)$ has compact resolvent $\forall z \in R$, where $C_{*}$ is as in (1.13).

The following theorem (see [15], p. 392 and p. 408) asserts the existence of sequences of eigenvalues and eigenvectors of the operator $A(\rho \hat{\eta})$ that are analytic on $\rho \in(-1,1)$ with values in $\mathbb{C}$ and $\mathbf{L}_{\#}^{2}(Y)$ respectively. That is, eigenvalues and eigenvectors can be expanded in Taylor's series in $\rho$, where the coefficients are in $\mathbb{C}$ and $\mathbf{L}_{\#}^{2}(Y)$ respectively. We refer to the eigenvalue curves and the eigenvector curves obtained in the above result as Rellich branches. For more details on Banach space valued holomorphic functions, we refer to [15,21].

Theorem 1.6 (Kato-Rellich). Let $A(z \hat{\eta})$ be a selfadjoint holomorphic family of type $(B)$ defined for $z$ in a neighbourhood $R$ in the complex plane of the interval $I:=(-1,1)$, with domains $\mathfrak{D}(A(z \hat{\eta}))$ contained in $\mathbf{L}_{\#}^{2}(Y)$. Furthermore, let $A(z \hat{\eta})+C_{*} I$ have compact resolvent for some $C_{*} \in \mathbb{R}$. Then, there exist a sequence of scalarvalued functions $\left\{\lambda_{\hat{\eta}, k}(\rho)\right\}_{k=1}^{\infty}$ and a sequence of $\mathbf{L}_{\#}^{2}(Y)$-valued functions $\left\{\boldsymbol{\Phi}_{\hat{\eta}, k}(\rho)\right\}_{k=1}^{\infty}$ defined on $I$ such that:

(i) For each fixed $\rho \in I$, the sequence $\left\{\lambda_{\hat{\eta}, k}(\rho)\right\}_{k=1}^{\infty}$ represents all the eigenvalues of $A(\rho \hat{\eta})$ including multiplicities (need not be a non-decreasing sequence) and the sequence $\left\{\boldsymbol{\Phi}_{\hat{\eta}, k}(\rho)\right\}_{k=1}^{\infty}$ represents corresponding eigenvectors.

(ii) For each $k \in \mathbb{N}$ and $\hat{\eta} \in \partial Y_{+}^{\prime}, \lambda_{\hat{\eta}, k}(\rho)$ and $\boldsymbol{\Phi}_{\hat{\eta}, k}(\rho)$ are analytic on I with values in $\mathbb{C}$ and $\mathbf{L}_{\#}^{2}(Y)$ respectively.

(iii) The sequence $\left\{\boldsymbol{\Phi}_{\hat{\eta}, k}(\rho)\right\}_{k=1}^{\infty}$ is an orthonormal basis for $\mathbf{L}_{\#}^{2}(Y)$.

Moreover, each of the elements of these eigenvalue-eigenvector sequences is holomorphic in a region $R_{\hat{\eta}, k}$ containing I of the complex plane, representing eigenelements of the operator $A(z \hat{\eta})$ for $z \in R_{\hat{\eta}, k}$.

Corollary 1.7. The Rellich branches can be re-indexed so that $\lambda_{\hat{\eta}, k}(0)=\mu_{k}(0)$ where $\left\{\mu_{k}(\eta)\right\}_{k=1}^{\infty}$ is the sequence of ordered eigenvalues given by Lemma 1.1 and, as a consequence, have the property

$$
\lambda_{\hat{\eta}, 1}(0) \leq \lambda_{\hat{\eta}, 2}(0) \leq \lambda_{\hat{\eta}, 3}(0) \leq \cdots \leq \lambda_{\hat{\eta}, k}(0) \leq \cdots
$$


Proof. This follows from the fact that each of the two sequences $\left\{\mu_{k}(\rho \hat{\eta})\right\}_{k=1}^{\infty}$ and $\left\{\lambda_{\hat{\eta}, k}(\rho)\right\}_{k=1}^{\infty}$ represents all the eigenvalues including multiplicities of $A(\rho \hat{\eta})$, for fixed $\hat{\eta} \in \partial Y_{+}^{\prime}$ and fixed $\rho \in I$.

Remark 1.8. Such a re-indexing as in the previous corollary can be done at any fixed $\rho_{0}$ but not for two values of $\rho$ simultaneously. Therefore, it may not be possible to re-index Rellich branches in such a way that the sequence of functions $\left\{\lambda_{\hat{\eta}, k}(.)\right\}_{k=1}^{\infty}$ is non-decreasing with $k \in \mathbb{N}$. Unlike eigenvalues, this re-indexing cannot be done with eigenvectors even at $\rho=0$. Also, $\boldsymbol{\Phi}_{\hat{\eta}, k}(0)$ cannot be prescribed in advance. To put it in Rellich's words, "the perturbation method itself selects them" (see [19]).

From the Theorem 1.6, we have for each $k \in \mathbb{N}, \boldsymbol{\Phi}_{\hat{\eta}, k}(\rho)$ is analytic on $I$ with values in $\mathbf{L}_{\#}^{2}(Y)$. As a consequence of the function $\left\|\boldsymbol{\Phi}_{\hat{\eta}, k}(.)\right\|_{\mathbf{H}_{\#}^{1}(Y)}$ being locally bounded (which is a consequence of the inequality (1.13)) and the density of $\mathbf{L}_{\#}^{2}(Y)$ in $\mathbf{H}_{\#}^{-1}(Y)$, it follows that the Rellich eigenvector branches are also analytic with values in $\mathbf{H}_{\#}^{1}(Y)$ (see p. 365 of [15]). We state this in the following lemma.

Lemma 1.9. Each of the Rellich eigenvector branches is analytic from $(-1,1)$ to $\mathbf{H}_{\#}^{1}(Y)$.

Remark 1.10. Not much can be said about the behaviour of the ordered eigenvalues $\left\{\mu_{k}(\eta), k=1,2,3\right\}$ near the origin. As seen below, in homogenization analysis, we will mainly deal with the directional spectrum $\left\{\lambda_{\hat{\eta}, k}(),. k \in \mathbb{N}\right\}$ introduced above. However, in Section 6, we present some properties of the ordered eigenvalues near the origin (see (6.10) in particular).

\section{Bloch WAVE DECOMPOSITION}

In this section we introduce an operator $A^{\epsilon}$, an $\epsilon$-scaled version of $A$. We then define Bloch waves and Bloch waves at $\epsilon$-scale associated with the operators $A$ and $A^{\epsilon}$ respectively and then prove that these waves give rise to a decomposition of the spaces $\mathbf{L}^{2}\left(\mathbb{R}^{3}\right), \mathbf{H}^{1}\left(\mathbb{R}^{3}\right)$ and $\mathbf{H}^{-1}\left(\mathbb{R}^{3}\right)$. As we shall see, the difference between the various cases lies in the nature of the convergence. Further, Parseval's equality holds only for $\mathbf{L}^{2}\left(\mathbb{R}^{3}\right)$; it is replaced by two inequalities in other cases (see (2.8), (2.24)).

First, we introduce the operator

$$
\left(A^{\epsilon} \mathbf{u}^{\epsilon}\right)_{p} \equiv-\frac{\partial}{\partial x_{q}}\left[\mathfrak{a}_{p q r s}^{\epsilon}(x) \mathfrak{e}_{r s}\left(\mathbf{u}^{\epsilon}(x)\right)\right] \equiv-\frac{\partial}{\partial x_{q}}\left[\mathfrak{a}_{p q r s}^{\epsilon}(x) \frac{\partial u_{r}^{\epsilon}}{\partial x_{s}}(x)\right], p=1,2,3,
$$

where the coefficients $\mathfrak{a}_{\text {pqrs }}^{\epsilon}(x)=\mathfrak{a}_{p q r s}\left(\frac{x}{\epsilon}\right)$ and $\mathfrak{a}_{p q r s}($.$) are as introduced in Section 1$ and satisfy (1.3), (1.4), and $\mathfrak{e}_{r s}($.$) as defined in (1.2). The associated shifted operator is given by$

$$
A^{\epsilon}(\xi)(\mathbf{u}) \stackrel{\text { def }}{=}-\left(\frac{\partial}{\partial x_{q}}+i \xi_{q}\right)\left\{\mathfrak{a}_{p q r s}\left(\frac{x}{\epsilon}\right) \frac{1}{2}\left[\left(\frac{\partial}{\partial x_{s}}+i \xi_{s}\right) u_{r}+\left(\frac{\partial}{\partial x_{r}}+i \xi_{r}\right) u_{s}\right]\right\} .
$$

\section{Definition of Bloch eigenvalues and Bloch eigenvectors}

Recall from Section 1.2 that, $\eta \in Y^{\prime}, \rho \in I$ and $\hat{\eta} \in \partial Y_{+}^{\prime}$ are related by the relations (1.11). We define two sequences of functions defined for almost every $\eta \in Y^{\prime}$ indexed by $m \in \mathbb{N}, \lambda_{m}(\eta)$ and $\boldsymbol{\Phi}_{m}(\eta)$, called Bloch eigenvalues and Bloch eigenvectors (Bloch waves) associated with the operator $A$ respectively in the following canonical way.

For non-zero $\eta \in Y^{\prime}$, we define

$$
\lambda_{m}(\eta):=\lambda_{\hat{\eta}, m}(\rho), \boldsymbol{\Phi}_{m}(y ; \eta):=\boldsymbol{\Phi}_{\hat{\eta}, m}(y ; \rho) .
$$


We now define the Bloch eigenvalues $\lambda_{m}^{\epsilon}(\xi)$ and Bloch eigenfunctions $\boldsymbol{\Phi}_{m}^{\epsilon}(. ; \xi) \in \mathbf{H}_{\#}^{1}(\epsilon Y)$, associated with the operator $A^{\epsilon}$ for $0<\epsilon \ll 1$ as follows: For non-zero $\xi \in \epsilon^{-1} Y^{\prime}$ and $x \in \mathbb{R}^{3}$, let

$$
\lambda_{m}^{\epsilon}(\xi):=\epsilon^{-2} \lambda_{m}(\epsilon \xi), \boldsymbol{\Phi}_{m}^{\epsilon}(x ; \xi):=\boldsymbol{\Phi}_{m}\left(\frac{x}{\epsilon} ; \epsilon \xi\right)
$$

where $\lambda_{m}($.$) and \boldsymbol{\Phi}_{m}(. ;$.$) are as introduced above. That is, \lambda_{m}^{\epsilon}(\xi)$ and $\boldsymbol{\Phi}_{m}^{\epsilon}(. ; \xi)$ are eigenvalue and eigenfunctions of the operator $A^{\epsilon}(\xi)$ The Bloch eigenvectors $\boldsymbol{\Phi}_{m}^{\epsilon}(\xi)$ are called Bloch waves at $\epsilon$-scale.

Owing to their definition $\lambda_{m}(\eta)$ and $\boldsymbol{\Phi}_{m}(\eta)$ have the following properties: the sequence $\left\{\lambda_{m}(\eta)\right\}_{m \in \mathbb{N}}$ may not be an increasing sequence for $\eta \neq 0$. The set $\left\{\boldsymbol{\Phi}_{m}(\eta): m \in \mathbb{N}\right\}$ forms an orthonormal basis for $\mathbf{L}_{\#}^{2}(Y)$ for each $0 \neq \eta \in Y^{\prime}$.

\subsection{Bloch wave decomposition of $\mathbf{L}^{2}\left(\mathbb{R}^{3}\right)$}

Bloch waves give rise to the following decomposition of the space of vector valued functions $\mathbf{L}^{2}\left(\mathbb{R}^{3}\right)$. Since the proof is classical (see, $[4,8,27]$ ), we do not repeat the same. The key idea in its proof is to use the fact that for $0 \neq \xi \in \epsilon^{-1} Y^{\prime},\left\{\epsilon^{-3 / 2} \mathbf{\Phi}_{m}^{\epsilon}(\xi): m \in \mathbb{N}\right\}$ forms an orthonormal basis for $\mathbf{L}_{\#}^{2}(\epsilon Y)$.

Theorem 2.1. Let $\mathbf{g}$ be an arbitrary element of the Hilbert space $\mathbf{L}^{2}\left(\mathbb{R}^{3}\right)$.

(i) For $m \in \mathbb{N}$, the following limit in $L^{2}\left(\epsilon^{-1} Y^{\prime}\right)$ exists, and is called the $m^{\text {th }}$ Bloch coefficient of $\mathbf{g}$ :

$$
\mathfrak{B}_{m}^{\epsilon} \mathbf{g}(\xi):=\lim _{R \rightarrow \infty} \int_{|x| \leq R} g_{p}(x) \mathrm{e}^{-i x \cdot \xi} \overline{\left(\mathbf{\Phi}_{m}^{\epsilon}\right)_{p}}(x ; \xi) \mathrm{d} x .
$$

(ii) Then the following inverse formula holds, where the limit is taken in the space $\mathbf{L}^{2}\left(\mathbb{R}^{3}\right)$ :

$$
\mathbf{g}(x)=\lim _{k \rightarrow \infty} \int_{\epsilon^{-1} Y^{\prime}} \sum_{m=1}^{k} \mathfrak{B}_{m}^{\epsilon} \mathbf{g}(\xi) \mathrm{e}^{i x . \xi} \boldsymbol{\Phi}_{m}^{\epsilon}(x ; \xi) \mathrm{d} \xi .
$$

(iii) Also, we have the following Plancheral identity: $\forall \mathbf{g}, \mathbf{h} \in \mathbf{L}^{2}\left(\mathbb{R}^{3}\right)$,

$$
\int_{\mathbb{R}^{3}} g_{p}(x) \bar{h}_{p}(x) \mathrm{d} x=\int_{\epsilon^{-1} Y^{\prime}} \mathfrak{B}_{m}^{\epsilon} \mathbf{g}(\xi) \overline{\mathfrak{B}_{m}^{\epsilon} \mathbf{h}(\xi)} \mathrm{d} \xi .
$$

We recall that the usual summation convention is followed in (2.4). This means that LHS is summed up with respect to $p=1,2,3$ and the RHS is summed up with respect to $m \in \mathbb{N}$. Above result says that we can identify the space $\mathbf{L}^{2}\left(\mathbb{R}^{3}\right)$ with $L^{2}\left(\epsilon^{-1} Y^{\prime} ; l^{2}(\mathbb{N})\right)$. Observe that, for each $m \in \mathbb{N}$, the $m$-th Bloch coefficient $\mathfrak{B}_{m}^{\epsilon} \mathbf{g}(\xi)$ defined above is scalar valued even though $\mathbf{g}$ is a vector valued function.

\subsection{Bloch wave decomposition of $\mathbf{H}^{1}\left(\mathbb{R}^{3}\right)$}

Following the analysis presented in the Section 1.2, the form $\mathbf{t}^{\epsilon}(\xi)$ corresponding to the operator $A^{\epsilon}(\xi)$ defined on the space $\mathbf{H}_{\#}^{1}(\epsilon Y)$ given by

$$
\begin{aligned}
\mathbf{t}^{\epsilon}(\xi)[\mathbf{u}, \mathbf{v}]= & \int_{\epsilon Y} \mathfrak{a}_{p q r s}\left(\frac{x}{\epsilon}\right) \frac{\partial u_{q}}{\partial x_{p}} \frac{\partial \overline{v_{r}}}{\partial x_{s}} \mathrm{~d} x+i \int_{\epsilon Y} \mathfrak{a}_{p q r s}\left(\frac{x}{\epsilon}\right)\left(\xi_{p} u_{q} \frac{\partial \overline{v_{r}}}{\partial x_{s}}-\xi_{s} \overline{v_{r}} \frac{\partial u_{q}}{\partial x_{p}}\right) \mathrm{d} x \\
& +\int_{\epsilon Y} \mathfrak{a}_{p q r s}\left(\frac{x}{\epsilon}\right) \xi_{p} \xi_{s} u_{q} \overline{v_{r}} \mathrm{~d} x
\end{aligned}
$$


satisfies, for some $C_{*}^{\epsilon}>\alpha / 4$,

$$
C_{1}\|\mathbf{g}\|_{\mathbf{H}_{\#}^{1}(\epsilon Y)}^{2} \leq \mathbf{t}^{\epsilon}(\xi)[\mathbf{g}, \mathbf{g}]+C_{*}^{\epsilon}(\mathbf{g}, \mathbf{g})_{\mathbf{L}_{\#}^{2}(\epsilon Y)} \leq C_{2} \epsilon^{-2}\|\mathbf{g}\|_{\mathbf{H}_{\#}^{1}(\epsilon Y)}^{2}
$$

for some positive constants $C_{1}, C_{2}$ independent of $\mathbf{g}$ and $\xi \in \epsilon^{-1} Y^{\prime}$. Thanks to the symmetries of the elastic tensor,

$$
\mathbf{t}^{\epsilon}(\xi)[., .]+C_{*}^{\epsilon}(., .) \mathbf{L}_{\#}^{2}(\epsilon Y)
$$

defines an inner product in $\mathbf{H}_{\#}^{1}(\epsilon Y)$.

With respect to the inner product (2.6), the family $\left\{\frac{\epsilon^{-3 / 2}}{\sqrt{C_{*}^{\epsilon}+\lambda_{m}^{\epsilon}(\xi)}} \mathbf{\Phi}_{m}^{\epsilon}(x ; \xi): m \in \mathbb{N}\right\}$ is an orthonormal basis for $\mathbf{H}_{\#}^{1}(\epsilon Y)$. This observation will now be used to obtain the following decomposition of $\mathbf{H}^{1}\left(\mathbb{R}^{3}\right)$.

Theorem 2.2. Let $\epsilon>0$ be fixed and $\mathbf{g}$ be an arbitrary element of $\mathbf{H}^{1}\left(\mathbb{R}^{3}\right)$.

(i) For $m \in \mathbb{N}$, we define the $m^{\text {th }}$ Bloch coefficient $\mathfrak{B}_{m}^{\epsilon} \mathbf{g}(\xi)$ by (2.2) with limit being taken in the weighted $L^{2}$ space with weight $\left(C_{*}^{\epsilon}+\lambda_{m}^{\epsilon}(\xi)\right)$ on $\epsilon^{-1} Y^{\prime}$.

(ii) Then the following inverse formula holds, where the limit is taken in the space $\mathbf{H}^{1}\left(\mathbb{R}^{3}\right)$ :

$$
\mathbf{g}(x)=\lim _{k \rightarrow \infty} \int_{\epsilon^{-1} Y^{\prime}} \sum_{m=1}^{k} \mathfrak{B}_{m}^{\epsilon} \mathbf{g}(\xi) \mathrm{e}^{i x \cdot \xi} \mathbf{\Phi}_{m}^{\epsilon}(x ; \xi) \mathrm{d} \xi .
$$

(iii) Also, we have the following inequalities:

$$
C_{1} \epsilon^{2}\|\mathbf{g}\|_{\mathbf{H}^{1}\left(\mathbb{R}^{3}\right)}^{2} \leq \int_{\epsilon^{-1} Y^{\prime}} \sum_{m=1}^{\infty}\left(C_{*}^{\epsilon}+\lambda_{m}^{\epsilon}(\xi)\right)\left|\mathfrak{B}_{m}^{\epsilon} \mathbf{g}(\xi)\right|^{2} \mathrm{~d} \xi \leq C_{2} \epsilon^{-4}\|\mathbf{g}\|_{\mathbf{H}^{1}\left(\mathbb{R}^{3}\right)}^{2},
$$

where $C_{1}, C_{2}$ are positive constants independent of $\mathbf{g}$ and $\epsilon$.

Remark 2.3. As is evident, one of the new features in Theorem 2.2 when compared with Theorem 2.1 is the stronger convergence in (2.7). The Parseval's equality for (2.4) is replaced by two inequalities in (2.8).

Proof of Theorem 2.2. By density arguments, we are reduced to the case of $\mathbf{g} \in \mathbf{H}^{1}\left(\mathbb{R}^{3}\right)$ having compact support in $\mathbb{R}^{3}$.

Step 1. The basic device of the proof is to associate periodic functions $\widetilde{\mathbf{g}}(. ; \xi) \in \mathbf{H}_{\#}^{1}(\epsilon Y)$ as follows:

$$
\widetilde{\mathbf{g}}(x ; \xi)=\sum_{\gamma \in 2 \pi \in \mathbb{Z}^{3}} \mathbf{g}(x+\gamma) \mathrm{e}^{-i(x+\gamma) \cdot \xi}
$$

Thanks to the observation preceding the statement of Theorem 2.2, we can write

$$
\widetilde{\mathbf{g}}(x ; \xi)=\sum_{m=1}^{\infty} c_{m}^{\epsilon}(\xi) \frac{\epsilon^{-3 / 2} \mathbf{\Phi}_{m}^{\epsilon}(x ; \xi)}{\sqrt{C_{*}^{\epsilon}+\lambda_{m}^{\epsilon}(\xi)}}
$$


where the convergence is in $\mathbf{H}_{\#}^{1}(\epsilon Y)$. Using the eigenvalue-eigenvector relation

$$
\mathbf{t}^{\epsilon}(\xi)\left[\mathbf{v}, \boldsymbol{\Phi}_{m}^{\epsilon}(\xi)\right]=\lambda_{m}^{\epsilon}(\xi)\left(\mathbf{v}, \boldsymbol{\Phi}_{m}^{\epsilon}(\xi)\right)_{\mathbf{L}_{\#}^{2}(\epsilon Y)}, \forall \mathbf{v} \in \mathbf{H}_{\#}^{1}(\epsilon Y)
$$

and $\epsilon$ Y-periodicity of $\boldsymbol{\Phi}_{m}^{\epsilon}(. ; \xi)$, the coefficient $c_{m}^{\epsilon}(\xi)$ can be easily calculated as

$$
c_{m}^{\epsilon}(\xi)=\epsilon^{-3 / 2} \sqrt{C_{*}^{\epsilon}+\lambda_{m}^{\epsilon}(\xi)} \int_{\mathbb{R}^{3}} g_{p}(x) \mathrm{e}^{-i x \cdot \xi} \overline{\left(\boldsymbol{\Phi}_{m}^{\epsilon}\right)_{p}}(x ; \xi) \mathrm{d} x,
$$

which by our definition, is equal to $\epsilon^{-3 / 2} \sqrt{C_{*}^{\epsilon}+\lambda_{m}^{\epsilon}(\xi)} \mathfrak{B}_{m}^{\epsilon} \mathbf{g}(\xi)$. Thus $(2.10)$ becomes

$$
\widetilde{\mathbf{g}}(x ; \xi)=\epsilon^{-3} \sum_{m=1}^{\infty} \mathfrak{B}_{m}^{\epsilon} \mathbf{g}(\xi) \boldsymbol{\Phi}_{m}^{\epsilon}(x ; \xi)
$$

Step 2 (Proof of inverse formula). To prove the inverse formula given in (2.7), we multiply both sides of the equation $(2.9)$ by $\mathrm{e}^{i x . \xi}$, and integrate with respect to $\xi \in \epsilon^{-1} Y^{\prime}$. Now, by observing that

$$
\int_{\epsilon^{-1} Y^{\prime}} \mathrm{e}^{-i \gamma \cdot \xi} \mathrm{d} \xi=0 \text { for } \gamma \neq 0
$$

we get

$$
\mathbf{g}(x)=\epsilon^{3} \int_{\epsilon^{-1} Y^{\prime}} \widetilde{\mathbf{g}}(x ; \xi) \mathrm{e}^{i x \cdot \xi} \mathrm{d} \xi .
$$

Using (2.11) in the above relation, we get

$$
\mathbf{g}(x)=\int_{\epsilon^{-1} Y^{\prime}} \sum_{m=1}^{\infty} \mathfrak{B}_{m}^{\epsilon} \mathbf{g}(\xi) \mathrm{e}^{i x . \xi} \boldsymbol{\Phi}_{m}^{\epsilon}(x ; \xi) \mathrm{d} \xi
$$

The relation (2.14) yields (2.7) by justifying the interchanging of the integral and the sum, which we do here. For each $k \in \mathbb{N}$, let $\mathbf{g}_{k} \in \mathbf{H}^{1}\left(\mathbb{R}^{3}\right)$ be defined by

$$
\mathbf{g}_{k}(x)=\int_{\epsilon^{-1} Y^{\prime}} \sum_{m=1}^{k} \mathfrak{B}_{m}^{\epsilon} \mathbf{g}(\xi) \mathrm{e}^{i x \cdot \xi} \boldsymbol{\Phi}_{m}^{\epsilon}(x ; \xi) \mathrm{d} \xi
$$

If we show that $\mathbf{g}_{k}$ is a Cauchy sequence in the space $\mathbf{H}^{1}\left(\mathbb{R}^{3}\right)$, the proof of the inverse formula will be finished.

Step 3 ( $\mathbf{g}_{k}$ is Cauchy in $\left.\mathbf{H}^{1}\left(\mathbb{R}^{3}\right)\right)$. Thanks to inequalities (2.8) whose proof will be given shortly, the real valued function $\|\mathbf{g}\|$ defined for elements $\mathbf{g} \in \mathbf{H}^{1}\left(\mathbb{R}^{3}\right)$ by

$$
\|\mathbf{g}\|^{2}:=\int_{Y^{\prime}} \sum_{m=1}^{\infty}\left(C_{*}^{\epsilon}+\lambda_{m}^{\epsilon}(\xi)\right)\left|\mathfrak{B}_{m}^{\epsilon} \mathbf{g}(\xi)\right|^{2} \mathrm{~d} \xi
$$


is an equivalent norm on $\mathbf{H}^{1}\left(\mathbb{R}^{3}\right)$. We prove that $\mathbf{g}_{k}$ is a Cauchy sequence in this norm. This follows easily by noting that for a.e. $\xi \in \epsilon^{-1} Y^{\prime}$, we have

$$
\mathfrak{B}_{m}^{\epsilon} \mathbf{g}_{k}(\xi)= \begin{cases}\mathfrak{B}_{m}^{\epsilon} \mathbf{g}(\xi) & \text { if } 1 \leq m \leq k \\ 0 & \text { if } m>k\end{cases}
$$

Step 4 (Proof of (2.8)). By Parseval theorem applied to (2.10), we have

$$
\mathbf{t}^{\epsilon}(\xi)[\widetilde{\mathbf{g}}(., \xi), \widetilde{\mathbf{g}}(., \xi)]+C_{*}^{\epsilon}(\widetilde{\mathbf{g}}(., \xi), \widetilde{\mathbf{g}}(., \xi))_{\mathbf{L}_{\#}^{2}(\epsilon Y)}=\epsilon^{-3} \sum_{m=1}^{\infty}\left(C_{*}^{\epsilon}+\lambda_{m}^{\epsilon}(\xi)\right)\left|\mathfrak{B}_{m}^{\epsilon} \mathbf{g}(\xi)\right|^{2}
$$

Therefore, for $\widetilde{\mathbf{g}}(., \xi)$ the inequalities $(2.5)$ read as

$$
C_{1}\|\widetilde{\mathbf{g}}(., \xi)\|_{\mathbf{H}_{\#}^{1}(\epsilon Y)}^{2} \leq \epsilon^{-3} \sum_{m=1}^{\infty}\left(C_{*}^{\epsilon}+\lambda_{m}^{\epsilon}(\xi)\right)\left|\mathfrak{B}_{m}^{\epsilon} \mathbf{g}(., \xi)\right|^{2} \leq C_{2} \epsilon^{-2}\|\widetilde{\mathbf{g}}(\xi)\|_{\mathbf{H}_{\#}^{1}(\epsilon Y)}^{2}
$$

Integrating the above inequality with respect to $\xi \in \epsilon^{-1} Y^{\prime}$, we get

$$
C_{1} \int_{\epsilon^{-1} Y^{\prime}}\|\widetilde{\mathbf{g}}(., \xi)\|_{\mathbf{H}_{\#}^{1}(\epsilon Y)}^{2} \mathrm{~d} \xi \leq \epsilon^{-3} \int_{\epsilon^{-1} Y^{\prime}} \sum_{m=1}^{\infty}\left(C_{*}^{\epsilon}+\lambda_{m}^{\epsilon}(\xi)\right)\left|\mathfrak{B}_{m}^{\epsilon} \mathbf{g}(\xi)\right|^{2} \mathrm{~d} \xi \leq C_{2} \epsilon^{-2} \int_{\epsilon^{-1} Y^{\prime}}\|\widetilde{\mathbf{g}}(., \xi)\|_{\mathbf{H}_{\#}^{1}(\epsilon Y)}^{2} \mathrm{~d} \xi .
$$

To complete the proof, it remains to estimate $\int_{\epsilon^{-1} Y^{\prime}}\|\widetilde{\mathbf{g}}(., \xi)\|_{\mathbf{H}_{\#}^{1}(\epsilon Y)}^{2} \mathrm{~d} \xi$. Now we will show that

$$
C_{3} \epsilon^{2}\|\mathbf{g}\|_{\mathbf{H}^{1}\left(\mathbb{R}^{3}\right)}^{2} \leq \epsilon^{3} \int_{\epsilon^{-1} Y^{\prime}}\|\widetilde{\mathbf{g}}(., \xi)\|_{\mathbf{L}_{\#}^{2}(\epsilon Y)}^{2} \mathrm{~d} \xi+\epsilon^{3} \sum_{j=1}^{3} \int_{\epsilon^{-1} Y^{\prime}}\left\|\frac{\partial \widetilde{\mathbf{g}}}{\partial x_{j}}(., \xi)\right\|_{\mathbf{L}_{\#}^{2}(\epsilon Y)}^{2} \mathrm{~d} \xi \leq C_{4} \epsilon^{-2}\|\mathbf{g}\|_{\mathbf{H}^{1}\left(\mathbb{R}^{3}\right)}^{2},
$$

where $C_{3}, C_{4}$ are positive constants. Combining (2.17) with (2.16), we see that the proof of (2.8) is finished.

Step 5 (Proof of (2.17)). Indeed by (2.9), we have

$$
\frac{\partial \widetilde{\mathbf{g}}}{\partial x_{j}}(x, \xi)=\frac{\widetilde{\partial \mathbf{g}}}{\partial x_{j}}(x ; \xi)-i \xi_{j} \widetilde{\mathbf{g}}(x ; \xi)
$$

It is classical that

$$
\epsilon^{3} \int_{\epsilon^{-1} Y^{\prime}}\|\widetilde{\mathbf{g}}(., \xi)\|_{\mathbf{L}_{\#}^{2}(\epsilon Y)}^{2} \mathrm{~d} \xi=\int_{\mathbb{R}^{3}}|\mathbf{g}(x)|^{2} \mathrm{~d} x, \quad \epsilon^{3} \int_{\epsilon^{-1} Y^{\prime}}\|\widetilde{\partial \mathbf{g}}(., \xi)\|_{\mathbf{L}_{\#}^{2}(\epsilon Y)}^{2} \mathrm{~d} \xi=\int_{\mathbb{R}^{3}}\left|\frac{\partial \mathbf{g}}{\partial x_{j}}(x)\right|^{2} \mathrm{~d} x .
$$


The second inequality in (2.17) follows from (2.19), in view of the relation (2.18). Also, we have (for each fixed $j=1,2,3)$

$$
\begin{aligned}
\left\|\frac{\partial \widetilde{\mathbf{g}}}{\partial x_{j}}(., \xi)\right\|_{\mathbf{L}_{\#}^{2}(\epsilon Y)}^{2} & \geq\left(\left|\xi_{j}\right|\|\widetilde{\mathbf{g}}(., \xi)\|_{\mathbf{L}_{\#}^{2}(\epsilon Y)}-\left\|\frac{\widetilde{\partial g}}{\partial x_{j}}(., \xi)\right\|_{\mathbf{L}_{\#}^{2}(\epsilon Y)}\right)^{2} \\
& =\left|\xi_{j}\right|^{2}\|\widetilde{\mathbf{g}}(., \xi)\|_{\mathbf{L}_{\#}^{2}(\epsilon Y)}^{2}+\left\|\frac{\partial \mathbf{g}}{\partial x_{j}}(., \xi)\right\|_{\mathbf{L}_{\#}^{2}(\epsilon Y)}^{2}-2\left|\xi_{j}\right|\|\widetilde{\mathbf{g}}(., \xi)\|_{\mathbf{L}_{\#}^{2}(\epsilon Y)}\left\|\widetilde{\frac{\partial \mathbf{g}}{\partial x_{j}}(., \xi)}\right\|_{\mathbf{L}_{\#}^{2}(\epsilon Y)} \\
& \geq\left\|\widetilde{\frac{\partial \mathbf{g}}{\partial x_{j}}}(., \xi)\right\|_{\mathbf{L}_{\#}^{2}(\epsilon Y)}^{2}-\epsilon^{-1}\|\widetilde{\mathbf{g}}(., \xi)\|_{\mathbf{L}_{\#}^{2}(\epsilon Y)}\left\|\frac{\partial \mathbf{g}}{\partial x_{j}}(., \xi)\right\|_{\mathbf{L}_{\#}^{2}(\epsilon Y)} \\
& \geq \frac{1}{2}\left\|\widetilde{\frac{\partial \mathbf{g}}{\partial x_{j}}}(., \xi)\right\|_{\mathbf{L}_{\#}^{2}(\epsilon Y)}^{2}-\frac{1}{2 \epsilon^{2}}\|\widetilde{\mathbf{g}}(., \xi)\|_{\mathbf{L}_{\#}^{2}(\epsilon Y)}^{2} .
\end{aligned}
$$

On summing up the above inequalities corresponding to $j=1,2,3$, and integrating on $\epsilon^{-1} Y^{\prime}$, we get the left inequality in (2.17).

Finally, we have an alternate expression for the inner product, given by,

$$
\mathbf{t}^{\epsilon}(\xi)[\mathbf{g}, \mathbf{h}]+C_{*}^{\epsilon}(\mathbf{g}, \mathbf{h})_{\mathbf{L}_{\#}^{2}(\epsilon Y)}=\sum_{m=1}^{\infty}\left(C_{*}^{\epsilon}+\lambda_{m}^{\epsilon}(\xi)\right)\left(\mathbf{g}, \epsilon^{-3 / 2} \mathbf{\Phi}_{m}^{\epsilon}\right)_{\mathbf{L}_{\#}^{2}(\epsilon Y)} \overline{\left(\mathbf{h}, \epsilon^{-3 / 2} \boldsymbol{\Phi}_{m}^{\epsilon}\right)_{\mathbf{L}_{\#}^{2}(\epsilon Y)},}
$$

for $\mathbf{g}, \mathbf{h} \in \mathbf{H}_{\#}^{1}(\epsilon Y)$, and follows on substituting the expansions for $\mathbf{g}$ and $\mathbf{h}$ in terms of Bloch waves in the LHS expression.

\subsection{Bloch wave decomposition of $\mathbf{H}^{-1}\left(\mathbb{R}^{3}\right)$}

We equip the space $\mathbf{H}_{\#}^{-1}(\epsilon Y)$ with the inner product

$$
\left[\mathbf{F}, \mathbf{F}^{\prime}\right]:=\sum_{m=1}^{\infty} \frac{1}{C_{*}^{\epsilon}+\lambda_{m}^{\epsilon}(\xi)} \overline{\left\langle\mathbf{F}, \epsilon^{-3 / 2} \mathbf{\Phi}_{m}^{\epsilon}\right\rangle}\left\langle\mathbf{F}^{\prime}, \epsilon^{-3 / 2} \mathbf{\Phi}_{m}^{\epsilon}\right\rangle
$$

where $<., .>$ denotes the duality product between $\mathbf{H}_{\#}^{-1}(\epsilon Y)$ and $\mathbf{H}_{\#}^{1}(\epsilon Y)$, which extends the $\mathbf{L}_{\#}^{2}(\epsilon Y)$-inner product. The corresponding norm is equivalent to the usual norm on $\mathbf{H}_{\#}^{-1}(\epsilon Y)$ and in fact, is equal to the norm of the dual space of $\mathbf{H}_{\#}^{1}(\epsilon Y)$ with the norm given by the inner product (2.6) (see [22]).

The family $\left\{\epsilon^{-3 / 2} \sqrt{C_{*}^{\epsilon}+\lambda_{m}^{\epsilon}(\xi)} \mathbf{\Phi}_{m}^{\epsilon}(x ; \xi): m \in \mathbb{N}\right\}$ forms an orthonormal basis for $\mathbf{H}_{\#}^{-1}(\epsilon Y)$, for each fixed $\xi \neq 0$ with respect to the inner product $(2.21)$. 
We now have the following result concerning the space $\mathbf{H}^{-1}\left(\mathbb{R}^{3}\right)$.

Theorem 2.4. Let $\epsilon>0$ be fixed and $\mathbf{F}=\mathbf{u}^{0}+\sum_{j=1}^{3} \frac{\partial}{\partial x_{j}} \mathbf{u}^{j} \in \mathbf{H}^{-1}\left(\mathbb{R}^{3}\right)$ be arbitrary.
(i) For $m \in \mathbb{N}$, we define the $m^{\text {th }}$ Bloch coefficient by

$$
\begin{aligned}
B_{m}^{\epsilon} \mathbf{F}(\xi):= & \lim _{R \rightarrow \infty} \int_{|x| \leq R} \mathrm{e}^{-i x \cdot \xi}\left\{\left(\mathbf{u}^{0}\right)_{p}(x) \overline{\left(\mathbf{\Phi}_{m}^{\epsilon}\right)_{p}}(x ; \xi)+i \sum_{j=1}^{3} \xi_{j}\left(\mathbf{u}^{j}\right)_{p}(x) \overline{\left(\mathbf{\Phi}_{m}^{\epsilon}\right)_{p}}(x ; \xi)\right\} \mathrm{d} x \\
& -\lim _{R \rightarrow \infty} \int_{|x| \leq R} \mathrm{e}^{-i x \cdot \xi} \sum_{j=1}^{3}\left(\mathbf{u}^{j}\right)_{p}(x) \frac{\partial \overline{\left(\mathbf{\Phi}_{m}^{\epsilon}\right)_{p}}}{\partial x_{j}}(x ; \xi) \mathrm{d} x,
\end{aligned}
$$

where the limit is taken in the weighted $L^{2}$ space with weight $\left(C_{*}^{\epsilon}+\lambda_{m}^{\epsilon}(\xi)\right)^{-1}$ on $\epsilon^{-1} Y^{\prime}$. Also, this definition is independent of the representation used for $\mathbf{F}$.

(ii) The following Plancheral identity holds: For $\mathbf{F} \in \mathbf{H}^{-1}\left(\mathbb{R}^{3}\right), \mathbf{g} \in \mathbf{H}^{1}\left(\mathbb{R}^{3}\right)$,

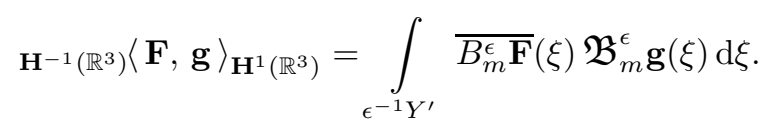

Thus, using the Bloch decomposition of $\mathbf{H}^{1}\left(\mathbb{R}^{3}\right)$ elements of Theorem 2.2, we see that $\mathbf{F} \in \mathbf{H}^{-1}\left(\mathbb{R}^{3}\right)$ may be expanded as

$$
\mathbf{F}(x)=\sum_{m=1}^{\infty} \int_{\epsilon^{-1} Y^{\prime}} B_{m}^{\epsilon} \mathbf{F}(\xi) \mathrm{e}^{i x \cdot \xi} \boldsymbol{\Phi}_{m}^{\epsilon}(x ; \xi) \mathrm{d} \xi
$$

(iii) Also, we have the following inequalities

$$
C_{4} \epsilon^{2}\|\mathbf{F}\|_{\mathbf{H}^{-1}\left(\mathbb{R}^{3}\right)}^{2} \leq \int_{\epsilon^{-1} Y^{\prime}} \sum_{m=1}^{\infty} \frac{\left|B_{m}^{\epsilon} \mathbf{F}(\xi)\right|^{2}}{C_{*}^{\epsilon}+\lambda_{m}^{\epsilon}(\xi)} \mathrm{d} \xi \leq C_{5} \epsilon^{-4}\|\mathbf{F}\|_{\mathbf{H}^{-1}\left(\mathbb{R}^{3}\right)}^{2},
$$

where $C_{4}, C_{5}$ are positive constants independent of $\mathbf{F}$ and $\epsilon$.

(iv) Also, for $\mathbf{F} \in \mathbf{H}^{1}\left(\mathbb{R}^{3}\right)$, we have $A^{\epsilon} \mathbf{F} \in \mathbf{H}^{-1}\left(\mathbb{R}^{3}\right)$ and

$$
B_{m}^{\epsilon}\left(A^{\epsilon} \mathbf{F}\right)(\xi)=\lambda_{m}^{\epsilon}(\xi) B_{m}^{\epsilon} \mathbf{F}(\xi), \forall m \in \mathbb{N} .
$$

Remark 2.5. There is nothing mysterious about the definition (2.22). It is formally obtained by a simple integration by parts in the definition (2.2) of $\mathfrak{B}_{m}^{\epsilon} \mathbf{F}(\xi)$. Further, it is obvious that $B_{m}^{\epsilon} \mathbf{F}(\xi)=\mathfrak{B}_{m}^{\epsilon} \mathbf{F}(\xi)$, if $\mathbf{F} \in \mathbf{L}^{2}\left(\mathbb{R}^{3}\right)$. Because of this, we see that the relation (2.23) is a generalization of (2.4) in the sense that if $\mathbf{F} \in \mathbf{L}^{2}\left(\mathbb{R}^{3}\right)$ then both (2.4) and (2.23) coincide.

Proof of Theorem 2.4. Let $\mathbf{F} \in \mathbf{H}^{-1}\left(\mathbb{R}^{3}\right)$ be given by

$$
\mathbf{F}=\mathbf{u}^{0}+\sum_{j=1}^{3} \frac{\partial}{\partial x_{j}} \mathbf{u}^{j}, \text { with } \mathbf{u}^{j} \in \mathbf{L}^{2}\left(\mathbb{R}^{3}\right), j=1,2,3 .
$$

We assume that $\mathbf{u}^{j}, j=0,1,2,3$, have compact support. Under this assumption, we prove the theorem. For general $\mathbf{F} \in \mathbf{H}^{-1}\left(\mathbb{R}^{3}\right)$, the theorem follows by the density of compactly supported functions in $\mathbf{L}^{2}\left(\mathbb{R}^{3}\right)$. 
Step 1. We now associate an element $\widetilde{\mathbf{F}}(. ; \xi) \in \mathbf{H}_{\#}^{-1}(\epsilon Y)$ for $\xi \in \epsilon^{-1} Y^{\prime}$ defined by

$$
\widetilde{\mathbf{F}}(x, \xi)=\widetilde{\mathbf{u}^{0}}(x, \xi)+\sum_{j=1}^{3} \widetilde{\frac{\partial}{\partial x_{j}}} \mathbf{u}^{j}(x, \xi)
$$

where we recall

$$
\begin{gathered}
\widetilde{\mathbf{u}^{j}}(x, \xi):=\sum_{\gamma \in 2 \pi \in \mathbb{Z}^{3}} \mathbf{u}^{j}(x+\gamma) \mathrm{e}^{-i(x+\gamma) \cdot \xi}, j=0,1,2,3 \\
\widetilde{\frac{\partial}{\partial x_{j}} \mathbf{u}^{j}(x, \xi)}:=\sum_{\gamma \in 2 \pi \in \mathbb{Z}^{3}} \frac{\partial}{\partial x_{j}} \mathbf{u}^{j}(x+\gamma) \mathrm{e}^{-i(x+\gamma) \cdot \xi}=\left(\frac{\partial}{\partial x_{j}}+i \xi_{j}\right) \widetilde{\mathbf{u}^{j}}(x, \xi), j=1,2,3 .
\end{gathered}
$$

Step 2. The map $\mathbf{F} \in \mathbf{H}^{-1}\left(\mathbb{R}^{3}\right) \longmapsto \widetilde{\mathbf{F}}(. ; \xi) \in \mathbf{H}_{\#}^{-1}(\epsilon Y)$ is well-defined. That is, though the definition of $\widetilde{\mathbf{F}}(. ; \xi)$ uses explicitly the given representation of $F \in \mathbf{H}^{-1}\left(\mathbb{R}^{3}\right)$, it is actually independent of the representation used. Let $\mathbf{F}_{1}$ and $\mathbf{F}_{2}$ be any two representations of $\mathbf{F}$ given by

$$
\mathbf{F}_{1}=\mathbf{u}^{0}+\sum_{j=1}^{3} \frac{\partial}{\partial x_{j}} \mathbf{u}^{j}, \quad \mathbf{F}_{2}=\mathbf{v}^{0}+\sum_{j=1}^{3} \frac{\partial}{\partial x_{j}} \mathbf{v}^{j} .
$$

We prove that $\widetilde{\mathbf{F}}_{1}(. ; \xi)=\widetilde{\mathbf{F}}_{2}(. ; \xi)$ as distributions. Since the equality is posed component-wise, we can assume that $\mathbf{u}^{j}, \mathbf{v}^{j}$ are scalar valued. That is, let $u_{0}, u_{1}, u_{2}, u_{3}$ and $v_{0}, v_{1}, v_{2}, v_{3}$ be scalar valued and satisfy $F=$ $u_{0}+\operatorname{div} \mathbf{u}=v_{0}+\operatorname{div} \mathbf{v}$ in $H^{-1}\left(\mathbb{R}^{3}\right)$, where $\mathbf{u}=\left(u_{1}, u_{2}, u_{3}\right), \mathbf{v}=\left(v_{1}, v_{2}, v_{3}\right)$. That is,

$$
\int_{\mathbb{R}^{3}}\left(\overline{u_{0}} \chi-\overline{u_{j}} \frac{\partial \chi}{\partial x_{j}}\right) \mathrm{d} x=\int_{\mathbb{R}^{3}}\left(\overline{v_{0}} \chi-\overline{v_{j}} \frac{\partial \chi}{\partial x_{j}}\right) \mathrm{d} x
$$

for every $\chi \in C_{0}^{\infty}\left(\mathbb{R}^{3}\right)$. We now compute $\widetilde{u_{0}}(., \xi)+\widetilde{\operatorname{div} \mathbf{u}}(., \xi) \in H_{\#}^{-1}(\epsilon Y)$ and find

$$
\widetilde{u_{0}}(x, \xi)+\widetilde{\operatorname{div} \mathbf{u}}(x, \xi)=\widetilde{u_{0}}(x, \xi)+\operatorname{div} \widetilde{\mathbf{u}}(x, \xi)+i \xi_{j} \widetilde{u_{j}}(x, \xi) .
$$

Simple calculations yield

$$
\left\langle\widetilde{u_{0}}(., \xi)+\operatorname{div} \widetilde{\mathbf{u}}(., \xi)+i \xi_{j} \widetilde{u_{j}}(., \xi), \chi\right\rangle=\left\langle\widetilde{v_{0}}(., \xi)+\operatorname{div} \widetilde{\mathbf{v}}(., \xi)+i \xi_{j} \widetilde{v_{j}}(., \xi), \chi\right\rangle
$$

Thus, we have proved $\widetilde{u_{0}}(., \xi)+\widetilde{\operatorname{div} \mathbf{u}}(., \xi)=\widetilde{v_{0}}(., \xi)+\widetilde{\operatorname{div} \mathbf{v}}(., \xi)$.

Step 3. We expand $\widetilde{\mathbf{F}}(x, \xi)$ in the orthonormal basis $\left\{\epsilon^{-3 / 2} \sqrt{C_{*}^{\epsilon}+\lambda_{m}^{\epsilon}(\xi)} \boldsymbol{\Phi}_{m}^{\epsilon}(x ; \xi): m \in \mathbb{N}\right\}$ of $\mathbf{H}_{\#}^{-1}(\epsilon Y)$ with respect to the inner product (2.21) as follows:

$$
\widetilde{\mathbf{F}}(x, \xi)=\sum_{m=1}^{\infty} c_{m}^{\epsilon}(\xi) \epsilon^{-3 / 2} \sqrt{C_{*}^{\epsilon}+\lambda_{m}^{\epsilon}(\xi)} \boldsymbol{\Phi}_{m}^{\epsilon}(x ; \xi)
$$

where the convergence of the series is in the space $\mathbf{H}_{\#}^{-1}(\epsilon Y)$ and

$$
c_{m}^{\epsilon}(\xi)=\left[\widetilde{\mathbf{F}}(., \xi), \epsilon^{-3 / 2} \sqrt{C_{*}^{\epsilon}+\lambda_{m}^{\epsilon}(\xi)} \boldsymbol{\Phi}_{m}^{\epsilon}(x ; \xi)\right] .
$$


We now compute and find that, in view of the definition $(2.22), c_{m}^{\epsilon}(\xi)$ is given by

$$
c_{m}^{\epsilon}(\xi)=\frac{\epsilon^{-3 / 2}}{\sqrt{C_{*}^{\epsilon}+\lambda_{m}^{\epsilon}(\xi)}} B_{m}^{\epsilon} \mathbf{F}(\xi) .
$$

Thus, the relation $(2.28)$ becomes

$$
\widetilde{\mathbf{F}}(x ; \xi)=\epsilon^{-3} \sum_{m=1}^{\infty} B_{m}^{\epsilon} \mathbf{F}(\xi) \boldsymbol{\Phi}_{m}^{\epsilon}(x ; \xi)
$$

Step 4 (Proof of Plancheral identity). We now have the following equality which follows via an easy computation of the RHS:

$$
\mathbf{H}^{-1}\left(\mathbb{R}^{3}\right)|\mathbf{F}, \mathbf{g}\rangle_{\mathbf{H}^{1}\left(\mathbb{R}^{3}\right)}=\int_{\epsilon^{-1} Y^{\prime}} \mathbf{H}_{\#}^{-1}(\epsilon Y)\left\langle\epsilon^{3 / 2} \widetilde{\mathbf{F}}(\xi), \epsilon^{3 / 2} \widetilde{\mathbf{g}}(\xi)\right\rangle_{\mathbf{H}_{\#}^{1}(\epsilon Y)} \mathrm{d} \xi,
$$

where $\mathbf{g}$ and $\widetilde{\mathbf{g}}$ are related by (2.13). Substituting in (2.32), the expressions for $\widetilde{\mathbf{F}}(x ; \xi)$ and $\widetilde{\mathbf{g}}(x ; \xi)$ from $(2.31)$ and (2.11) respectively, we get the Plancheral identity (2.23).

Step 5 (Proof of (2.24)). From the Plancheral identity (2.23) and (2.8), we get the inequalities (2.24) by duality.

Step 6 (Proof of (2.25)). Since the operator $A^{\epsilon}$ is in the divergence form, it follows that $A^{\epsilon} \mathbf{F}$ belongs to $\mathbf{H}^{-1}\left(\mathbb{R}^{3}\right)$ for every $\mathbf{F} \in \mathbf{H}^{1}\left(\mathbb{R}^{3}\right)$. By $(2.26)$, clearly $\widetilde{A^{\epsilon}} \mathbf{F}(x ; \xi)=A^{\epsilon}(\xi) \widetilde{\mathbf{F}}(x ; \xi)$. By the definition of $B_{m}^{\epsilon}\left(A^{\epsilon} \mathbf{F}\right)$ (see (2.29) and (2.30)), we have

$$
\begin{aligned}
B_{m}^{\epsilon}\left(A^{\epsilon} \mathbf{F}\right)(\xi) & =\left(C_{*}^{\epsilon}+\lambda_{m}^{\epsilon}(\xi)\right)\left[A^{\epsilon}(\xi) \widetilde{\mathbf{F}}(., \xi), \boldsymbol{\Phi}_{m}^{\epsilon}(. ; \xi)\right]=\overline{\left\langle A^{\epsilon}(\xi) \widetilde{\mathbf{F}}(., \xi), \boldsymbol{\Phi}_{m}^{\epsilon}(. ; \xi)\right\rangle} \\
& =\overline{\left\langle\widetilde{\mathbf{F}}(., \xi), A^{\epsilon}(\xi) \mathbf{\Phi}_{m}^{\epsilon}(. ; \xi)\right\rangle}=\lambda_{m}^{\epsilon}(\xi) \overline{\left\langle\widetilde{\mathbf{F}}(., \xi), \boldsymbol{\Phi}_{m}^{\epsilon}(. ; \xi)\right\rangle}=\lambda_{m}^{\epsilon}(\xi) B_{m}^{\epsilon} \mathbf{F}(\xi) .
\end{aligned}
$$

Remark 2.6 (a comment on the inner products used). We employed the inner product given by (2.6) for the space $\mathbf{H}_{\#}^{1}(\epsilon Y)$. Indeed this inner product (2.6) arises from the bilinear forms associated with shifted operators $A^{\epsilon}(\xi)$. The space $\mathbf{H}_{\#}^{-1}(\epsilon Y)$, which is the dual space of $\mathbf{H}_{\#}^{1}(\epsilon Y)$, was then given the inner product (2.21).

The norm of Bloch eigenvectors in the spaces $\mathbf{H}_{\#}^{1}(\epsilon Y)$, measured with respect to the inner product (2.6), is related to the corresponding Bloch eigenvalue. As a consequence the norm of eigenvectors in the dual space $\mathbf{H}_{\#}^{-1}(\epsilon Y)$ is reciprocal to the norm measured in (2.6). This approach was convenient for us in proving Theorems 2.2 and 2.4.

The following result provides the crucial estimates uniform with respect to $\epsilon$. We do not prove it here and we refer the reader to a similar estimates in the scalar case (Lems. 2.1 and 2.2 in [6]).

\section{Lemma 2.7 .}

(i) There exist positive constants $\alpha_{1}$ and $\alpha_{2}$ (independent of $\epsilon$ ) such that

$$
\alpha_{1}\|\mathbf{g}\|_{\mathbf{H}^{1}\left(\mathbb{R}^{3}\right)}^{2} \leq \int_{\epsilon^{-1} Y^{\prime}} \sum_{m=1}^{\infty}\left(1+\lambda_{m}^{\epsilon}(\xi)\right)\left|B_{m}^{\epsilon} \mathbf{g}(\xi)\right|^{2} \mathrm{~d} \xi \leq \alpha_{2}\|\mathbf{g}\|_{\mathbf{H}^{1}\left(\mathbb{R}^{3}\right)}^{2}, \forall \mathbf{g} \in \mathbf{H}^{1}\left(\mathbb{R}^{3}\right) .
$$

(ii) There exist positive constants $\beta_{1}$ and $\beta_{2}$ (independent of $\epsilon$ ) such that

$$
\beta_{1}\|\mathbf{F}\|_{\mathbf{H}^{-1}\left(\mathbb{R}^{3}\right)}^{2} \leq \int_{\epsilon^{-1} Y^{\prime}} \sum_{m=1}^{\infty} \frac{\left|B_{m}^{\epsilon} \mathbf{F}(\xi)\right|^{2}}{1+\lambda_{m}^{\epsilon}(\xi)} \mathrm{d} \xi \leq \beta_{2}\|\mathbf{F}\|_{\mathbf{H}^{-1}\left(\mathbb{R}^{3}\right)}^{2}, \forall \mathbf{F} \in \mathbf{H}^{-1}\left(\mathbb{R}^{3}\right) .
$$




\section{Computations with Bloch spectrum}

In this section, we make connections between Bloch space and physical space. The test functions $\mathbf{w}^{\text {rs }}$ defined by the cell problem (3.2), the homogenized coefficients given by (3.1) naturally appear in these conditions and we recall them here. The classical homogenized coefficients $\mathfrak{b}_{\text {pqrs }}$ are given by (see, for instance, [4, 18, 23])

$$
\mathfrak{b}_{\text {pqrs }}=\frac{1}{|Y|} \int_{Y} \mathfrak{a}_{\text {pqrs }}(y) \mathrm{d} y+\frac{1}{|Y|} \int_{Y} \mathfrak{a}_{p q l m}(y) \mathfrak{e}_{l m}\left(\mathbf{w}^{r s}(y)\right) \mathrm{d} y
$$

where $\mathbf{w}^{r s} \in \mathbf{H}_{\#}^{1}(Y)$ solves the following cell problem:

$$
\left(A \mathbf{w}^{r s}\right)_{p} \equiv-\frac{\partial}{\partial y_{q}}\left(\mathfrak{a}_{p q l m}(y) \mathfrak{e}_{l m}\left(\mathbf{w}^{r s}\right)\right)=\frac{\partial \mathfrak{a}_{p q r s}(y)}{\partial y_{q}} \quad \text { in } Y, p,=1,2,3 .
$$

We already noted (see Rem. 1.8) that $\boldsymbol{\Phi}_{\hat{\eta}, m}(0)$ cannot be prescribed in advance and the perturbation itself selects them. This natural selection follows a rule (see (3.5) below).

The following result is concerning the relations between the first and second order derivatives of eigenvalues $\lambda_{\hat{\eta}, m}(\rho)$, and first order derivatives of eigenvectors $\boldsymbol{\Phi}_{\hat{\eta}, m}(\rho)$. In the calculations that follow, we suppress the dependence on $y$ in $\boldsymbol{\Phi}_{\hat{\eta}, m}(y ; \rho)$, for $m=1,2,3$, for convenience, and denote by $\boldsymbol{\Phi}_{\hat{\eta}, m}(\rho)$; the prime denotes the derivative with respect to $\rho$.

Lemma 3.1. For $m=1,2,3$, and for fixed $\hat{\eta} \in \partial Y_{+}^{\prime}$, we have:

(i) $\lambda_{\hat{\eta}, m}(0)=0$, and corresponding eigenvector $\boldsymbol{\Phi}_{\hat{\eta}, m}(0)$ is independent of $y$.

(ii) The eigenvalue $\lambda_{\hat{\eta}, m}(\rho)$ has a critical point at $\rho=0$. That is,

$$
\lambda_{\hat{\eta}, m}^{\prime}(0)=0 .
$$

(iii) The derivative of the eigenvector $\boldsymbol{\Phi}_{\hat{\eta}, m}(\rho)$ at $\rho=0$ satisfies:

$$
\boldsymbol{\Phi}_{\hat{\eta}, m}^{\prime}(0)-i \hat{\eta}_{s} \mathbf{w}^{r s}(y)\left(\boldsymbol{\Phi}_{\hat{\eta}, m}(0)\right)_{r}
$$

is a constant vector in $\mathbb{C}^{3}$ i.e., independent of $y$, where $\mathbf{w}^{r s}, r, s=1,2,3$ solves the cell problem (3.2).

(iv) The second derivative of the eigenvalue $\lambda_{\hat{\eta}, m}(\rho)$ at $\rho=0$ satisfies the relation

$$
\frac{1}{2} \lambda_{\hat{\eta}, m}^{\prime \prime}(0) \boldsymbol{\Phi}_{\hat{\eta}, m}(0)=M(\hat{\eta} ; \mathfrak{b}) \boldsymbol{\Phi}_{\hat{\eta}, m}(0)
$$

where $M(\hat{\eta} ; \mathfrak{b})$ is a matrix whose $(p, r)$-th entry $M_{p r}(\hat{\eta} ; \mathfrak{b})$ is given by $M_{p r}(\hat{\eta} ; \mathfrak{b})=\mathfrak{b}_{\text {pqrs }} \hat{\eta}_{q} \hat{\eta}_{s}$, and $\mathfrak{b}_{\text {pqrs }}$ are the classical homogenized coefficients as given in (3.1). The above relation is referred to as propagation condition.

Proof. We use that for $\boldsymbol{\Phi}_{\hat{\eta}, m}(\rho) \in \mathbf{H}_{\#}^{1}(Y)$ is an eigenvector of the operator $A(\rho \hat{\eta})$ corresponding to the eigenvalue $\lambda_{\hat{\eta}, m}(\rho)$; that is

$$
A(\rho \hat{\eta}) \boldsymbol{\Phi}_{\hat{\eta}, m}(\rho)=\lambda_{\hat{\eta}, m}(\rho) \boldsymbol{\Phi}_{\hat{\eta}, m}(\rho) \text { in } Y
$$

which reads as

$$
-\left(\frac{\partial}{\partial y_{q}}+i \rho \hat{\eta}_{q}\right)\left\{\mathfrak{a}_{p q r s}(y)\left(\frac{\partial}{\partial y_{s}}+i \rho \hat{\eta}_{s}\right)\left(\boldsymbol{\Phi}_{\hat{\eta}, m}\right)_{r}(\rho)\right\}=\lambda_{\hat{\eta}, m}(\rho)\left(\boldsymbol{\Phi}_{\hat{\eta}, m}\right)_{p}(\rho), p=1,2,3
$$


We write the above equation as

$$
\left(A^{0}+\rho A^{1}+\rho^{2} A^{2}\right)\left(\boldsymbol{\Phi}_{\hat{\eta}, m}(\rho)\right)=\lambda_{\hat{\eta}, m}(\rho) \boldsymbol{\Phi}_{\hat{\eta}, m}(\rho),
$$

where the operators $A^{0}, A^{1}, A^{2}$ formally acting on vector functions $\mathbf{u}=\mathbf{u}(y)$, are given by

$$
\begin{aligned}
& \left(A^{0} \mathbf{u}\right)_{p}=-\frac{\partial}{\partial y_{q}}\left(\mathfrak{a}_{p q r s}(y) \frac{\partial u_{r}}{\partial y_{s}}\right), \\
& \left(A^{1} \mathbf{u}\right)_{p}=-i \hat{\eta}_{s} \frac{\partial}{\partial y_{q}}\left(\mathfrak{a}_{p q r s}(y) u_{r}\right)-i \hat{\eta}_{q}\left(\mathfrak{a}_{p q r s}(y) \frac{\partial u_{r}}{\partial y_{s}}\right), \\
& \left(A^{2} \mathbf{u}\right)_{p}=\mathfrak{a}_{p q r s}(y) \hat{\eta}_{q} \hat{\eta}_{s} u_{r}, \quad p=1,2,3 .
\end{aligned}
$$

Step 1 (Proof of (3.3)). Differentiating (3.7) with respect to $\rho$, we get

$$
\begin{array}{r}
\left\{A(\rho \hat{\eta})\left(\boldsymbol{\Phi}_{\hat{\eta}, m}^{\prime}(\rho)\right)\right\}_{p}+\left(A^{1}\left(\boldsymbol{\Phi}_{\hat{\eta}, m}(\rho)\right)\right)_{p}+2 \rho \mathfrak{a}_{p q r s} \hat{\eta}_{q} \hat{\eta}_{s}\left(\boldsymbol{\Phi}_{\hat{\eta}, m}(\rho)\right)_{r}= \\
\lambda_{\hat{\eta}, m}^{\prime}(\rho) \boldsymbol{\Phi}_{\hat{\eta}, m}(\rho)+\lambda_{\hat{\eta}, m}(\rho) \boldsymbol{\Phi}_{\hat{\eta}, m}^{\prime}(\rho) .
\end{array}
$$

Evaluating the equation (3.8) at $\rho=0$, and taking $\mathbf{L}^{2}(Y)$-innerproduct with $\boldsymbol{\Phi}_{\hat{\eta}, m}(0)$, and integrating by parts we get

$$
\lambda_{\hat{\eta}, m}^{\prime}(0)=\left(A^{1}\left(\boldsymbol{\Phi}_{\hat{\eta}, m}(0)\right), \boldsymbol{\Phi}_{\hat{\eta}, m}(0)\right)_{\mathbf{L}^{2}(Y)}=0,
$$

by using the structure of the operator $A^{1}$ and noting that $\boldsymbol{\Phi}_{\hat{\eta}, m}(0)$ is independent of $y$ for $m=1,2,3$. This proves (3.3).

Step 2 (Proof of (3.4)). From (3.3) and (3.8), we get

$$
A^{0}\left(\boldsymbol{\Phi}_{\hat{\eta}, m}^{\prime}(0)\right)=-A^{1}\left(\boldsymbol{\Phi}_{\hat{\eta}, m}(0)\right)=-i \hat{\eta}_{s} \frac{\partial}{\partial y_{q}}\left(\mathfrak{a}_{p q r s}(y)\right)\left(\boldsymbol{\Phi}_{\hat{\eta}, m}(0)\right)_{r} .
$$

Recalling that $\mathbf{w}^{r s}(y)$ solves the cell problem (3.2), we get (3.4).

Step 3 (Proof of (3.5)). Differentiating (3.8) with respect to $\rho$, we get

$$
\left.\begin{array}{r}
{\left[\left(A(\rho \hat{\eta})-\lambda_{\hat{\eta}, m}(\rho)\right) \boldsymbol{\Phi}_{\hat{\eta}, m}^{\prime \prime}(\rho)\right]_{p}+\left[A^{1}\left(\boldsymbol{\Phi}_{\hat{\eta}, m}^{\prime}(\rho)\right)\right]_{p}+4 \rho \mathfrak{a}_{p q r s} \hat{\eta}_{q} \hat{\eta}_{s}\left(\boldsymbol{\Phi}_{\hat{\eta}, m}^{\prime}(\rho)\right)_{r}} \\
+2 \mathfrak{a}_{p q r s} \hat{\eta}_{q} \hat{\eta}_{s}\left(\boldsymbol{\Phi}_{\hat{\eta}, m}(\rho)\right)_{r}-i \hat{\eta}_{s} \frac{\partial}{\partial y_{q}}\left(\mathfrak{a}_{p q r s}(y)\left(\boldsymbol{\Phi}_{\hat{\eta}, m}^{\prime}(\rho)\right)_{r}\right) \\
-i \hat{\eta}_{q} \mathfrak{a}_{p q r s} \frac{\partial}{\partial y_{s}}\left(\boldsymbol{\Phi}_{\hat{\eta}, m}^{\prime}(\rho)\right)_{r}-2 \lambda_{\hat{\eta}, m}^{\prime}(\rho)\left[\boldsymbol{\Phi}_{\hat{\eta}, m}^{\prime}(\rho)\right]_{p}=\lambda_{\hat{\eta}, m}^{\prime \prime}(\rho)\left[\boldsymbol{\Phi}_{\hat{\eta}, m}(\rho)\right]_{p}, p=1,2,3 .
\end{array}\right\}
$$

We first evaluate the equation (3.10) at $\rho=0$, and then take $\mathbf{L}^{2}(Y)$-innerproduct with the constant vector $\boldsymbol{\Phi}_{\hat{\eta}, m}(0) \in \mathbb{C}^{3}$. We then integrate the resultant expression over $Y$. Using the observation that derivative of a periodic function integrates to zero on the basic periodic cell, We see that only the fourth and sixth terms on the left hand side of (3.10) have non-zero contribution and this involves the classical homogenized coefficients $\mathfrak{b}_{\text {pqrs }}$. This follows from the relations (3.3) and (3.4). Thus we get (3.5).

\subsection{Few remarks on propagation condition}

In this paragraph we highlight the differences between the scalar case of [8] and the present case of systems with respect to the propagation condition. Propagation condition determines uniquely the homogenized coefficients. This is more direct in the scalar case but is somewhat implicit in the case of systems. We also explain the reason behind the nomenclature "propagation condition" for the relation (3.5). 
Remark 3.2 (comparison with scalar case). In the scalar case (see [8]) the propagation condition involves only $m=1$ since $\lambda_{1}(0)$ is a simple eigenvalue of $A(0)$. Also $\lambda_{\hat{\eta}, 1}^{\prime \prime}(0)$ is quadratic in $\hat{\eta}$ due to the fact that $\lambda_{1}(\eta)$ is analytic in the variable $\eta$ near $\eta=0$. Since $\boldsymbol{\Phi}_{\hat{\eta}, 1}(0)$ is a non-zero scalar (being an eigenvector of $A(0)$ ), we cancel out $\boldsymbol{\Phi}_{\hat{\eta}, 1}(0)$ in the relation (3.5) to get an equality of two quadratic forms: the quadratic form associated with the Hessien of $\lambda_{1}(\eta)$ at $\eta=0$ and the one corresponding to the homogenized coefficients $\mathfrak{b}_{k l}$. It is well-known that the homogenized matrix $\left(\mathfrak{b}_{k l}\right)$ is symmetric and therefore we get the equality of matrices $\left(\frac{1}{2} \frac{\partial^{2} \lambda_{1}}{\partial \eta_{k} \partial \eta_{l}}(0)\right)=\left(\mathfrak{b}_{k l}\right)$ since they give rise to the same quadratic form.

In the present case of elasticity system, there are two striking differences compared to the scalar case. They are as follows:

(i) We cannot cancel $\boldsymbol{\Phi}_{\hat{\eta}, m}(0)$ as they are vectors. In fact the propagation condition is an eigenvalue relation for the matrix $M(\hat{\eta})$.

(ii) Even though $M(\hat{\eta})$ depends quadratically on $\hat{\eta}, \frac{1}{2} \lambda_{\hat{\eta}, m}^{\prime \prime}(0), m=1,2,3$ need not be quadratic in $\hat{\eta}$.

However the relations (3.5) are enough to obtain the homogenized elasticity system.

\section{Lemma 3.3.}

(i) The propagation condition characterizes the homogenized coefficients. That is, if there is a tensor $\mathfrak{c}_{\text {pqrs }}$ with the same symmetries as that of $\mathfrak{b}_{\text {pqrs }}$ satisfying the propagation condition then $\mathfrak{c}_{\text {pqrs }}=\mathfrak{b}_{\text {pqrs }}$.

(ii) The numbers $\sqrt{\frac{1}{2} \lambda_{\hat{\eta}, m}^{\prime \prime}(0)}, m=1,2,3$, are the speeds of propagation of waves propagating in the homogenized medium in the direction $\hat{\eta}$.

Proof.

Proof of (i). If $\mathfrak{c}_{\text {pqrs }}$ has symmetries (1.3) and satisfies the propagation condition with $\mathfrak{c}_{\text {pqrs }}$ replacing $\mathfrak{b}_{\text {pqrs }}$, then clearly $M(\hat{\eta} ; \mathfrak{b})=M(\hat{\eta} ; \mathfrak{c})$ i.e., $\mathfrak{c}_{\text {pqrs }} \hat{\eta}_{q} \hat{\eta}_{s}=\mathfrak{b}_{\text {pqrs }} \hat{\eta}_{q} \hat{\eta}_{s}$ for every $p$ and $r$ since the action of two matrices $M(\hat{\eta} ; \mathfrak{b})$ and $M(\hat{\eta} ; \mathfrak{c})$ is the same on a basis for $\mathbb{C}^{3}$. This implies that $\mathfrak{b}_{\text {pqrs }}-\mathfrak{c}_{\text {pqrs }}$ is skew-symmetric in $q, s$, for each fixed $p, r$. Let us denote $\mathfrak{d}_{\text {pqrs }} \equiv \mathfrak{b}_{\text {pqrs }}-\mathfrak{c}_{\text {pqrs }}$. From the symmetries of $\mathfrak{b}_{\text {pqrs }}, \mathfrak{c}_{\text {pqrs }}$, we get $\mathfrak{d}_{\text {pqrs }}$ also satisfies the same symmetries (1.4). We also have from our observation, $\mathfrak{d}_{\text {pqrs }}=-\mathfrak{d}_{\text {psrq }}$. These observations are enough to conclude that $\mathfrak{d}_{\text {pqrs }}=0$ for every $p, q, r, s=1,2,3$.

Proof of (ii). Consider the following elastic-wave equation in the homogenized medium:

$$
\mathbf{u}_{t t}=\frac{\partial}{\partial x_{q}}\left(\mathfrak{b}_{\text {pqrs }} \mathfrak{e}_{r s}(\mathbf{u}(x))\right)
$$

We look for travelling wave solutions of (3.11) which are of the form $\mathbf{u}(x, t) \equiv f(\hat{\eta} \cdot x-c t) \mathbf{L}$. Clearly, $\mathbf{u}(x, t) \equiv$ $f(\hat{\eta} . x-c t) \mathbf{L}$ is a solution of (3.11) if and only if

$$
c^{2} \mathbf{L}=M(\hat{\eta} ; \mathfrak{b}) \mathbf{L}
$$

where $M(\hat{\eta} ; \mathfrak{b})$ is a matrix whose (p,r)-th entry $M_{p r}(\hat{\eta} ; \mathfrak{b})$ is given by $M_{p r}(\hat{\eta} ; \mathfrak{b})=\mathfrak{b}_{p q r s} \hat{\eta}_{q} \hat{\eta}_{s}$. Clearly (ii) follows on comparing (3.12) and (3.5).

\section{Interpretation}

In the context of wave propagation in a homogeneous medium, it is customary to seek solutions of the form

$$
\mathbf{u}(x, t)=f(\hat{\eta} . x-c t) \mathbf{L} .
$$

From this representation, it is clear that $\hat{\eta}$ represents the direction of propagation; $c$, the speed; $\mathbf{L}$, the polarization vector. From the relations (3.12) and (3.5), we can say that $\boldsymbol{\Phi}_{\hat{\eta}, m}(0)(m=1,2,3)$ are polarization vectors 
and $\sqrt{\frac{1}{2} \lambda_{\hat{\eta}, m}^{\prime \prime}(0)}(m=1,2,3)$ are speeds of waves propagating in the direction $\hat{\eta}$. This conclusion was possible because of our approach of fixing a direction $\hat{\eta}$ and looking for directional regularity of spectral elements. Recall that we were led into this approach in order to overcome certain difficulties involved in multi-parameter problems.

\section{Asymptotic Behaviour of the first three Bloch transforms}

In the scalar case treated in [8], it was shown that the first Bloch transform converges to Fourier transform. This can be interpreted as saying that the periodic medium approaches a homogeneous medium. In the case of systems, such a result is, strictly speaking, not valid. What is true is the following lemma which is precisely what is needed in the passage to the limit carried out in Section 5.

Lemma 4.1. Let $\mathbf{g}, \mathbf{g}^{\epsilon} \in \mathbf{L}^{2}\left(\mathbb{R}^{3}\right)$ be such that the support of $\mathbf{g}^{\epsilon}$ is contained in a fixed compact subset $K \subseteq \mathbb{R}^{3}$,

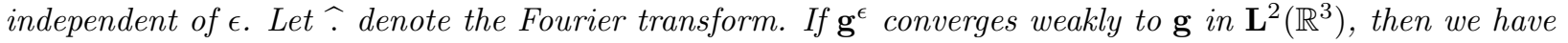

$$
\chi_{\epsilon^{-1} Y^{\prime}}(\xi) B_{m}^{\epsilon} \mathbf{g}^{\epsilon}(\xi) \rightarrow|Y|^{1 / 2} \overline{\left(\mathbf{\Phi}_{\hat{\eta}, m}\right)_{p}}(0) \widehat{g_{p}}(\xi)
$$

in $L_{\text {loc }}^{2}\left(\mathbb{R}_{\xi}^{3}\right)$-weak, for $m=1,2,3$, where we recall that $\hat{\eta}$ depends on $\xi$ and in fact $\xi$ and $\hat{\eta} \in \partial Y_{+}^{\prime}$ are related by the relation $\hat{\eta}=\frac{1}{2 \xi_{l}} \xi$, where $l$ is the least index such that $\xi_{l} \neq 0$.

Proof.

Step 1. From the definition of $B_{m}^{\epsilon} \mathbf{g}^{\epsilon}(\xi)$, we have

$$
B_{m}^{\epsilon} \mathbf{g}^{\epsilon}(\xi)=\int_{\mathbb{R}^{3}} \mathrm{e}^{-i x \cdot \xi} g_{p}^{\epsilon}(x) \overline{\left(\boldsymbol{\Phi}_{m}^{\epsilon}\right)_{p}}(x ; \xi) \mathrm{d} x=\int_{\mathbb{R}^{3}} \mathrm{e}^{-i x \cdot \xi} g_{p}^{\epsilon}(x) \overline{\left(\boldsymbol{\Phi}_{m}\right)_{p}}\left(\frac{x}{\epsilon} ; \epsilon \xi\right) \mathrm{d} x
$$

We add and subtract $\mathrm{e}^{-i x \cdot \xi} g_{p}^{\epsilon}(x) \overline{\left(\boldsymbol{\Phi}_{\hat{\eta}, m}\right)_{p}}\left(\frac{x}{\epsilon} ; 0\right)$ to the integrand above, and this yields

$$
\left.\begin{array}{l}
\chi_{\epsilon^{-1} Y^{\prime}}(\xi) B_{m}^{\epsilon} \mathbf{g}^{\epsilon}(\xi)=\chi_{\epsilon^{-1} Y^{\prime}}(\xi) \int_{K} \mathrm{e}^{-i x \cdot \xi} g_{p}^{\epsilon}(x) \overline{\left(\mathbf{\Phi}_{\hat{\eta}, m}\right)_{p}}\left(\frac{x}{\epsilon} ; 0\right) \mathrm{d} x \\
\quad+\chi_{\epsilon^{-1} Y^{\prime}}(\xi) \int_{K} \mathrm{e}^{-i x \cdot \xi} g_{p}^{\epsilon}(x)\left(\overline{\left(\boldsymbol{\Phi}_{\hat{\eta}, m}\right)_{p}}\left(\frac{x}{\epsilon} ; \epsilon \frac{(\xi \cdot \hat{\eta})}{|\hat{\eta}|^{2}}\right)-\overline{\left(\boldsymbol{\Phi}_{\hat{\eta}, m}\right)_{p}}\left(\frac{x}{\epsilon} ; 0\right)\right) \mathrm{d} x
\end{array}\right\}
$$

Step 2. The second term on the right hand side of (4.2) is estimated using Cauchy-Schwarz inequality as follows:

$$
\begin{aligned}
& \left|\chi_{\epsilon^{-1} Y^{\prime}}(\xi) \int_{K} \mathrm{e}^{-i x \cdot \xi} g_{p}^{\epsilon}(x)\left(\overline{\left(\boldsymbol{\Phi}_{\hat{\eta}, m}\right)_{p}}\left(\frac{x}{\epsilon} ; \epsilon \frac{(\xi \cdot \hat{\eta})}{|\hat{\eta}|^{2}}\right)-\overline{\left(\boldsymbol{\Phi}_{\hat{\eta}, m}\right)_{p}}\left(\frac{x}{\epsilon} ; 0\right)\right) \mathrm{d} x\right| \\
& \leq\left\|\mathbf{g}^{\epsilon}(x)\right\|_{\mathbf{L}^{2}(K)}\left(\int_{K}\left|\left(\boldsymbol{\Phi}_{\hat{\eta}, m}\right)_{p}\left(\frac{x}{\epsilon} ; \epsilon \frac{(\xi \cdot \hat{\eta})}{|\hat{\eta}|^{2}}\right)-\left(\boldsymbol{\Phi}_{\hat{\eta}, m}\right)_{p}\left(\frac{x}{\epsilon} ; 0\right)\right|^{2} \mathrm{~d} x\right)^{1 / 2} \\
& \leq c\left\|\boldsymbol{\Phi}_{\hat{\eta}, m}\left(y ; \epsilon \frac{(\xi . \hat{\eta})}{|\hat{\eta}|^{2}}\right)-\mathbf{\Phi}_{\hat{\eta}, m}(y ; 0)\right\|_{\mathbf{L}^{2}(Y)},
\end{aligned}
$$


where $c$ depends on the measure of the compact set $K$, and on the bound of $\left\|g^{\epsilon}(x)\right\|_{\mathbf{L}^{2}(K)}$ but independent of $\xi$. The RHS of the inequality (4.3) is bounded by $2 c$, since by our normalization of eigenvectors, we have

$$
\left\|\boldsymbol{\Phi}_{\hat{\eta}, m}\left(y ; \epsilon \frac{(\xi \cdot \hat{\eta})}{|\hat{\eta}|^{2}}\right)\right\|_{\mathbf{L}^{2}(Y)}=\left\|\boldsymbol{\Phi}_{\hat{\eta}, m}(0)\right\|_{\mathbf{L}^{2}(Y)}=1
$$

and converges to 0 as $\epsilon \rightarrow 0$, for each fixed $\xi$ since $\boldsymbol{\Phi}_{\hat{\eta}, m}(\rho)$ depends continuously on $\rho$ with values in $\mathbf{L}_{\#}^{2}(Y)$. Now, applying the dominated convergence theorem, we see that the second term on the right hand side of (4.2) goes to zero in $L_{l o c}^{2}\left(\mathbb{R}^{3}\right)$.

Step 3. Since $\boldsymbol{\Phi}_{\hat{\eta}, m}(0)$ is independent of $x$, the first term on the right hand side of (4.2) is equal to

$$
\chi_{\epsilon^{-1} Y^{\prime}}(\xi)|Y|^{1 / 2} \overline{\left(\Phi_{\hat{\eta}, m}\right)_{p}}(0) \widehat{g_{p}^{\epsilon}}(\xi) .
$$

As the Fourier transform operator is weakly continuous on $L^{2}\left(\mathbb{R}^{3}\right)$, we get $\widehat{g_{p}^{\epsilon}} \rightarrow \widehat{g_{p}}$ in $L^{2}\left(\mathbb{R}_{\xi}^{3}\right)$-weak. As a consequence, the first term on the RHS of (4.2) converges in $L^{2}\left(\mathbb{R}_{\xi}^{3}\right)$-weak to

$$
|Y|^{1 / 2} \overline{\left(\Phi_{\hat{\eta}, m}\right)_{p}}(0) \widehat{g_{p}}(\xi) \text {. }
$$

This finishes the proof of (4.1).

Also, in view of the following pointwise convergence of the sequence $\left(\sqrt{1+\lambda_{m}^{\epsilon}(\xi)}\right)^{-1}$ which is uniformly bounded by 1 :

$$
\left(\sqrt{1+\lambda_{m}^{\epsilon}(\xi)}\right)^{-1} \longrightarrow\left(\sqrt{1+\frac{(\xi \cdot \hat{\eta})^{2}}{2|\hat{\eta}|^{4}} \lambda_{\hat{\eta}, m}^{\prime \prime}(0)}\right)^{-1}
$$

As a consequence, the above convergence holds in $L_{l o c}^{2}\left(\mathbb{R}_{\xi}^{3}\right)$ as well. Combining this observation with Lemma 4.2, we obtain the following result.

Corollary 4.2. For $m=1,2,3$, under the hypotheses and in the notations of Lemma 4.1, we have the following convergence in $L_{\text {loc }}^{2}\left(\mathbb{R}_{\xi}^{3}\right)$-weak:

$$
\chi_{\epsilon^{-1} Y^{\prime}}(\xi) \frac{B_{m}^{\epsilon} \mathbf{g}^{\epsilon}(\xi)}{\sqrt{1+\lambda_{m}^{\epsilon}(\xi)}} \rightarrow \frac{|Y|^{1 / 2} \overline{\left(\mathbf{\Phi}_{\hat{\eta}, m}\right)_{p}}(0)}{\sqrt{1+\frac{(\xi \cdot \hat{\eta})^{2}}{2|\hat{\eta}|^{4}}} \lambda_{\hat{\eta}, m}^{\prime \prime}(0)} \widehat{g}_{p}(\xi)
$$

\section{Homogenization RESUlt AND its PROOF}

In this section we state the classical homogenization result for the three dimensional linear elasticity system and prove it via Bloch wave method. We recall that the tensor $\mathfrak{b}_{\text {pqrs }}$ is already defined in the Section 3 by (3.1). We now state the homogenization result.

Theorem 5.1 (homogenization result). Let $\Omega$ be an arbitrary domain in $\mathbb{R}^{3}$ and $\mathbf{f} \in \mathbf{L}^{2}(\Omega)$. Let $\mathbf{u}^{\epsilon} \in \mathbf{H}^{1}(\Omega)$, $\mathbf{u}^{*} \in \mathbf{H}^{1}(\Omega)$ be such that $\mathbf{u}^{\epsilon}$ converges weakly to $\mathbf{u}^{*}$ in $\mathbf{H}^{1}(\Omega)$, and

$$
A^{\epsilon} \mathbf{u}^{\epsilon}=\mathbf{f} \text { in } \Omega \text {. }
$$


Then

(i) We have the following convergence of stresses:

$$
\mathfrak{a}_{p q r s}\left(\frac{x}{\epsilon}\right) \mathfrak{e}_{r s}\left(\mathbf{u}^{\epsilon}(x)\right) \rightarrow \mathfrak{b}_{p q r s} \mathfrak{e}_{r s}\left(\mathbf{u}^{*}(x)\right) \text { in } L^{2}(\Omega)-\text { weak, } \forall p, q=1,2,3 .
$$

(ii) The limit $\mathbf{u}^{*} \in \mathbf{H}^{1}(\Omega)$ satisfies homogenized system of equations:

$$
\left(A^{*} \mathbf{u}^{*}\right)_{p} \equiv-\frac{\partial}{\partial x_{q}}\left[\mathfrak{b}_{p q r s} \mathfrak{e}_{r s}\left(\mathbf{u}^{*}(x)\right)\right]=f_{p} \quad \text { in } \Omega, \quad p=1,2,3 .
$$

(iii) Moreover, the tensor $\left(\mathfrak{b}_{\text {pqrs }}\right)$ has the symmetries: $\mathfrak{b}_{\text {qprs }}=\mathfrak{b}_{\text {pqrs }}=\mathfrak{b}_{\text {rspq }}=\mathfrak{b}_{\text {pqsr }}$.

The (5.2) above is called the homogenized system corresponding to the problem (5.1).

We now turn to the proof of Theorem 5.1. We indicate an outline of the proof. The first step is to localize the equation (5.1) which is in $\Omega$ to get an equation in $\mathbb{R}^{3}$. We obtain a set of relations in Bloch space indexed by $m \in \mathbb{N}$ which is equivalent to the localized equation by taking Bloch coefficients of the localized equation. We then pass to the limit in these relations corresponding to $m=1,2,3$. The limiting system can be thought of as the homogenized system in the Fourier space. An application of inverse Fourier transform yields the homogenized system.

Finally, we recall that in the scalar case (see [8]), the first eigenmode plays the dominant role. Its counterpart in the case of system is the set of three Bloch modes, which are indexed by $m=1,2,3$.

\subsection{Localization}

Let $\psi_{0}$ be a fixed element in $\mathcal{D}(\Omega)$ with support $K$. Suppose $\mathbf{u}^{\epsilon}$ satisfy $A^{\epsilon} \mathbf{u}^{\epsilon}=\mathbf{f}$ in $\Omega$ and bounded in $\mathbf{H}^{1}(\Omega)$. Then, $\psi_{0} \mathbf{u}^{\epsilon}$ satisfies:

$$
\begin{gathered}
A^{\epsilon}\left(\psi_{0} \mathbf{u}^{\epsilon}\right)(x)=\psi_{0} \mathbf{f}(x)+\mathbf{g}^{\epsilon}(x)+\mathbf{h}^{\epsilon}(x) \text { in } \mathbb{R}^{3}, \\
\text { where } g_{p}^{\epsilon}(x):=-\frac{\partial \psi_{0}}{\partial x_{q}}(x) a_{p q r s}^{\epsilon}(x) \frac{\partial u_{r}^{\epsilon}}{\partial x_{s}}(x), p=1,2,3, \\
h_{p}^{\epsilon}(x):=-\frac{\partial}{\partial x_{q}}\left(\frac{\partial \psi_{0}}{\partial x_{s}}(x) a_{p q r s}^{\epsilon}(x) u_{r}^{\epsilon}(x)\right), p=1,2,3 .
\end{gathered}
$$

Note that $\mathbf{g}^{\epsilon}$ is bounded in $\mathbf{L}^{2}\left(\mathbb{R}^{3}\right)$, and $\mathbf{h}^{\epsilon}$ is bounded only in $\mathbf{H}^{-1}\left(\mathbb{R}^{3}\right)$. By Theorem 2.4, the localized equation (5.3) is equivalent to the set of relations: For $\xi \in \epsilon^{-1} Y^{\prime}$ a.e., $m \in \mathbb{N}$,

$$
\lambda_{m}^{\epsilon}(\xi) B_{m}^{\epsilon}\left(\psi_{0} \mathbf{u}^{\epsilon}\right)(\xi)=B_{m}^{\epsilon}\left(\psi_{0} \mathbf{f}\right)(\xi)+B_{m}^{\epsilon} \mathbf{g}^{\epsilon}(\xi)+B_{m}^{\epsilon} \mathbf{h}^{\epsilon}(\xi)
$$

The relation (5.6) follows from the relation (2.25).

\subsection{Limit of $B_{m}^{\epsilon} \mathbf{g}^{\epsilon}$}

We will apply the Corollary 4.2 with $\mathbf{g}^{\epsilon}$ given by (5.4), which is bounded in $\mathbf{L}^{2}\left(\mathbb{R}^{3}\right)$, and hence has a subsequence weakly converging to $\mathbf{g}^{*}$ in $\mathbf{L}^{2}\left(\mathbb{R}^{3}\right)$. This subsequence (still denoted by $\epsilon$ ) satisfies the hypothesis of the Corollary 4.2. We will compute $\mathbf{g}^{*}$ now. Since the sequence $\sigma_{p q}^{\epsilon}(x):=a_{p q r s}^{\epsilon}(x) \frac{\partial u_{r}^{\epsilon}}{\partial x_{s}}(x)$ is bounded in $L^{2}(\Omega)$, there exists a subsequence which converges weakly in $L^{2}(\Omega)$, which we denote by $\sigma_{p q}^{*}$ for all $p, q=1,2,3$. We extend $\sigma_{p q}^{*}$ by zero outside $\Omega$, and we still denote the extension by $\sigma_{p q}^{*}$. Thus, we see that $\mathbf{g}^{*}$ is given by (from (5.4))

$$
g_{p}^{*}(x)=-\sigma_{p q}^{*}(x) \frac{\partial \psi_{0}}{\partial x_{q}}(x), p=1,2,3
$$


Thus, by Corollary 4.2 we have

$$
\chi_{\epsilon^{-1} Y^{\prime}}(\xi) \frac{B_{m}^{\epsilon} \mathbf{g}^{\epsilon}(\xi)}{\sqrt{1+\lambda_{m}^{\epsilon}(\xi)}} \rightarrow \frac{-|Y|^{1 / 2} \overline{\left(\mathbf{\Phi}_{\hat{\eta}, m}\right)_{p}}(0)}{\sqrt{1+\frac{(\xi . \hat{\eta})^{2}}{2|\hat{\eta}|^{4}} \lambda_{\hat{\eta}, m}^{\prime \prime}(0)}}\left(\sigma_{p q}^{*}(x) \overline{\frac{\partial \psi_{0}}{\partial x_{q}}}(x)\right)(\xi)
$$

in $L_{\text {loc }}^{2}\left(\mathbb{R}_{\xi}^{3}\right)$-weak, for $m=1,2,3$.

\subsection{Limit of $B_{m}^{\epsilon} \mathbf{h}^{\epsilon}$}

Regarding the asymptotic behaviour of $B_{m}^{\epsilon} \mathbf{h}^{\epsilon}, m=1,2,3$, we have the following lemma.

Lemma 5.2. Let $\mathbf{h}^{\epsilon}$ be defined by (5.5). For $m=1,2,3$, we have the following convergence in $L_{\text {loc }}^{2}\left(\mathbb{R}_{\xi}^{3}\right)$-weak:

$$
\chi_{\epsilon^{-1} Y^{\prime}}(\xi) \frac{B_{m}^{\epsilon} \mathbf{h}^{\epsilon}(\xi)}{\sqrt{1+\lambda_{m}^{\epsilon}(\xi)}} \rightarrow-\frac{-i|Y|^{1 / 2} \overline{\left(\mathbf{\Phi}_{\hat{\eta}, m}\right)_{p}}(0)}{\sqrt{1+\frac{(\xi \cdot \hat{\eta})^{2}}{2|\hat{\eta}|^{4}} \lambda_{\hat{\eta}, m}^{\prime \prime}(0)}} \xi_{q} \mathfrak{b}_{p q r s}\left(\frac{\partial \psi_{0}}{\partial x_{s}}(x) u_{r}^{*}(x)\right)
$$

Proof.

Step 1. By the definition of the Bloch transform (see Th. 2.4 for elements of $\mathbf{H}^{-1}\left(\mathbb{R}^{3}\right)$ ), we have

$$
\begin{aligned}
\frac{B_{m}^{\epsilon} \mathbf{h}^{\epsilon}(\xi)}{\sqrt{1+\lambda_{m}^{\epsilon}(\xi)}}= & -\frac{i \xi_{q}}{\sqrt{1+\lambda_{m}^{\epsilon}(\xi)}} \int_{\mathbb{R}^{3}} \mathrm{e}^{-i x \cdot \xi} a_{p q r s}^{\epsilon}(x) \frac{\partial \psi_{0}}{\partial x_{s}}(x) u_{r}^{\epsilon}(x) \overline{\left(\mathbf{\Phi}_{\hat{\eta}, m}\right)_{p}}\left(\frac{x}{\epsilon} ; \epsilon \frac{(\xi \cdot \hat{\eta})}{|\hat{\eta}|^{2}}\right) \mathrm{d} x \\
& +\frac{1}{\sqrt{1+\lambda_{m}^{\epsilon}(\xi)}} \int_{\mathbb{R}^{3}} \mathrm{e}^{-i x \cdot \xi} \mathfrak{a}_{p q r s}\left(\frac{x}{\epsilon}\right) \frac{\partial \psi_{0}}{\partial x_{s}}(x) u_{r}^{\epsilon}(x) \frac{\partial \overline{\left(\boldsymbol{\Phi}_{\hat{\eta}, m}\right)_{p}}}{\partial x_{q}}\left(\frac{x}{\epsilon} ; \epsilon \frac{(\xi \cdot \hat{\eta})}{|\hat{\eta}|^{2}}\right) \mathrm{d} x .
\end{aligned}
$$

Step 2. Denoting by $l_{p}(x):=-i \xi_{q} a_{p q r s}^{\epsilon}(x) \frac{\partial \psi_{0}}{\partial x_{s}}(x) u_{r}^{\epsilon}(x), p=1,2,3$, the first integral on the right hand side of (5.9) is equal to $\left(\sqrt{1+\lambda_{m}^{\epsilon}(\xi)}\right)^{-1} B_{m}^{\epsilon} \mathbf{l}^{\epsilon}(\xi)$. Applying Corollary 4.2 to $\mathbf{l}^{\epsilon}$, we get

$$
\chi_{\epsilon^{-1} Y^{\prime}}(\xi) \frac{B_{m}^{\epsilon} \mathbf{l}^{\epsilon}(\xi)}{\sqrt{1+\lambda_{m}^{\epsilon}(\xi)}} \rightarrow \frac{-i \xi_{q}|Y|^{1 / 2} \mathcal{M}_{Y}\left(\mathfrak{a}_{p q r s}\right) \overline{\left(\mathbf{\Phi}_{\hat{\eta}, m}\right)_{p}}(0)}{\sqrt{1+\frac{(\xi \cdot \hat{\eta})^{2}}{2|\hat{\eta}|^{4}} \lambda_{\hat{\eta}, m}^{\prime \prime}(0)}}\left(\frac{\partial \psi_{0}}{\partial x_{s}}(x) u_{r}^{*}(x)\right)(\xi)
$$

in $L_{\text {loc }}^{2}\left(\mathbb{R}^{3}\right)$-weak, for $m=1,2,3$.

Step 3. We now find limit of the second term on the right hand side of (5.9) as follows. In this step, we use the directional regularity of the Bloch modes $i . e$. , the analyticity of $\boldsymbol{\Phi}_{\hat{\eta}, m}(y ; \rho), m=1,2,3$; with respect to the parameter $\rho$ near zero. We have the following Taylor series expansion in $H_{\#}^{1}(Y)$ of $\boldsymbol{\Phi}_{\hat{\eta}, m}(y ; \rho)$ near $\rho=0$ :

$$
\boldsymbol{\Phi}_{\hat{\eta}, m}(y ; \rho)=\boldsymbol{\Phi}_{\hat{\eta}, m}(y ; 0)+\rho \mathbf{\Phi}_{\hat{\eta}, m}^{\prime}(y ; 0)+\gamma_{\hat{\eta}, m}(y ; \rho)
$$

Since $\gamma_{\hat{\eta}, m}(y ; 0)=\frac{\partial \gamma_{\hat{\eta}, m}}{\partial \rho}(y ; 0)=0$, we have $\gamma_{\hat{\eta}, m}(. ; \rho)=O\left(|\rho|^{2}\right)$ in $L^{\infty}\left(I ; H_{\#}^{1}(Y)\right)$. We also have $\frac{\partial \gamma_{\hat{\eta}, m}}{\partial y_{k}}(. ; \rho)=$ $O\left(|\rho|^{2}\right)$ in $L^{\infty}\left(I ; L_{\#}^{2}(Y)\right)$. 
Substituting $y=x / \epsilon$ and $\eta=\epsilon \xi$ in (5.11), we get

$$
\boldsymbol{\Phi}_{\hat{\eta}, m}\left(\frac{x}{\epsilon} ; \epsilon \frac{(\xi . \hat{\eta})}{|\hat{\eta}|^{2}}\right)=\boldsymbol{\Phi}_{\hat{\eta}, m}\left(\frac{x}{\epsilon} ; 0\right)+\epsilon \frac{(\xi . \hat{\eta})}{|\hat{\eta}|^{2}} \boldsymbol{\Phi}_{\hat{\eta}, m}^{\prime}\left(\frac{x}{\epsilon} ; 0\right)+\gamma_{\hat{\eta}, m}\left(\frac{x}{\epsilon} ; \epsilon \frac{(\xi . \hat{\eta})}{|\hat{\eta}|^{2}}\right) .
$$

Differentiating the last expression with respect to $x_{k}$ yields

$$
\frac{\partial}{\partial x_{k}} \boldsymbol{\Phi}_{\hat{\eta}, m}\left(\frac{x}{\epsilon} ; \epsilon \frac{(\xi \cdot \hat{\eta})}{|\hat{\eta}|^{2}}\right)=\frac{(\xi \cdot \hat{\eta})}{|\hat{\eta}|^{2}} \frac{\partial}{\partial y_{k}} \boldsymbol{\Phi}_{\hat{\eta}, m}^{\prime}\left(\frac{x}{\epsilon} ; 0\right)+\epsilon^{-1} \frac{\partial \gamma_{\hat{\eta}, m}}{\partial y_{k}}\left(\frac{x}{\epsilon} ; \epsilon \frac{(\xi . \hat{\eta})}{|\hat{\eta}|^{2}}\right) .
$$

For $\xi$ belonging to the set $\{\xi: \epsilon \xi \in U$ and $|\xi| \leq M\}$, we have

$$
\frac{\partial \gamma_{\hat{\eta}, m}}{\partial y_{k}}\left(. ; \epsilon \frac{(\xi \cdot \hat{\eta})}{|\hat{\eta}|^{2}}\right)=O\left(\left|\epsilon \frac{(\xi \cdot \hat{\eta})}{|\hat{\eta}|^{2}}\right|^{2}\right)=\epsilon^{2} O\left(|\xi|^{2}\right) \leq C M^{2} \epsilon^{2} .
$$

This implies that

$$
\epsilon^{-2} \frac{\partial \gamma_{\hat{\eta}, m}}{\partial y_{k}}\left(\frac{x}{\epsilon} ; \epsilon \frac{(\xi \cdot \hat{\eta})}{|\hat{\eta}|^{2}}\right) \in L_{l o c}^{\infty}\left(\mathbb{R}_{\xi}^{N} ; L_{\#}^{2}(\epsilon Y)\right) .
$$

The second term on the RHS of (5.9) is given by

$$
\begin{aligned}
& \chi_{\epsilon^{-1} Y^{\prime}}(\xi) \frac{B_{m}^{\epsilon}\left(\mathbf{h}^{\epsilon}-\mathbf{l}^{\epsilon}\right)(\xi)}{\sqrt{1+\lambda_{m}^{\epsilon}(\xi)}}= \\
& \quad \frac{\chi_{\epsilon^{-1} Y^{\prime}}(\xi)}{\sqrt{1+\lambda_{m}^{\epsilon}(\xi)}}\left\{\int_{K} \mathrm{e}^{-i x \cdot \xi} \mathfrak{a}_{p q r s}\left(\frac{x}{\epsilon}\right) \frac{\partial \psi_{0}}{\partial x_{s}}(x) u_{r}^{\epsilon}(x) \frac{\partial}{\partial x_{q}}\left(\overline{\mathbf{\Phi}_{\hat{\eta}, m}}\left(\frac{x}{\epsilon} ; \epsilon \frac{(\xi \cdot \hat{\eta})}{|\hat{\eta}|^{2}}\right)\right)_{p} \mathrm{~d} x\right\} .
\end{aligned}
$$

Using the Taylor expansion of $\boldsymbol{\Phi}_{\hat{\eta}, m}(y ; \rho)$ near $\rho=0$, the RHS of (5.13) becomes

$$
\frac{\chi_{\epsilon^{-1} Y^{\prime}}(\xi)}{\sqrt{1+\lambda_{m}^{\epsilon}(\xi)}} \epsilon^{-1} \int_{\mathbb{R}^{3}} \mathrm{e}^{-i x \cdot \xi} \frac{\partial \psi_{0}}{\partial x_{s}}(x) \mathfrak{a}_{p q r s}\left(\frac{x}{\epsilon}\right) u_{r}^{\epsilon}(x) \frac{\partial}{\partial y_{q}}\left[\epsilon \frac{(\xi \cdot \hat{\eta})}{|\hat{\eta}|^{2}} \overline{\Phi_{\hat{\eta}, m}^{\prime}}\left(\frac{x}{\epsilon} ; 0\right)+\gamma_{\hat{\eta}, m}\left(\frac{x}{\epsilon} ; \epsilon \frac{(\xi \cdot \hat{\eta})}{|\hat{\eta}|^{2}}\right)\right]_{p} \cdot(
$$

In view of (5.12), the quantity (5.14) converges pointwise to

$$
\mathcal{M}_{Y}\left(\mathfrak{a}_{p q r s}(y) \frac{\partial}{\partial y_{q}}\left(\overline{\Phi_{\hat{\eta}, m}^{\prime}}(y ; 0)\right)_{p}\right) \frac{(\xi \cdot \hat{\eta})|\hat{\eta}|^{-2}}{\sqrt{1+\frac{(\xi \cdot \hat{\eta})^{2}}{2|\hat{\eta}|^{4}} \lambda_{\hat{\eta}, m}^{\prime \prime}(0)}} \int_{\mathbb{R}^{3}} \frac{\partial \psi_{0}}{\partial x_{s}}(x) \mathrm{e}^{-i x \cdot \xi} u_{r}^{*}(x),
$$

and hence it is also its $L_{l o c}^{2}\left(\mathbb{R}_{\xi}^{3}\right)$-weak limit.

Step 4. Calculation of $\mathcal{M}_{Y}\left(\mathfrak{a}_{p q r s}(y) \frac{\partial}{\partial y_{q}}\left(\overline{\Phi_{\hat{\eta}, m}^{\prime}}(y ; 0)\right)_{p}\right)$

From Lemma 3.1, we have

$$
\mathbf{\Phi}_{\hat{\eta}, m}^{\prime}(y ; 0)-i \hat{\eta}_{s} \mathbf{w}^{r s}(y)\left(\boldsymbol{\Phi}_{\hat{\eta}, m}(y ; 0)\right)_{r}
$$

is a constant vector in $\mathbb{C}^{3}$, where $\mathbf{w}^{r s}, r, s=1,2,3$ solves the cell problem (3.2).

Hence we have

$$
\frac{\partial}{\partial y_{q}}\left(\boldsymbol{\Phi}_{\hat{\eta}, m}^{\prime}(y ; 0)\right)_{p}=i \hat{\eta}_{s} \frac{\partial}{\partial y_{q}}\left(\mathbf{w}^{r s}\right)_{p}(y)\left(\mathbf{\Phi}_{\hat{\eta}, m}(y ; 0)\right)_{r} .
$$


Therefore, we have

$$
\mathcal{M}_{Y}\left(\mathfrak{a}_{p q r s}(y) \frac{\partial}{\partial y_{q}}\left(\overline{\mathbf{\Phi}_{\hat{\eta}, m}^{\prime}}(y ; 0)\right)_{p}\right)=-i \hat{\eta}_{l} \mathcal{M}_{Y}\left(\mathfrak{a}_{p q r s}(y) \frac{\partial}{\partial y_{q}}\left(\mathbf{w}^{k l}\right)_{p}(y)\right) \overline{\left(\boldsymbol{\Phi}_{\hat{\eta}, m}\right)_{k}}(0)
$$

Step 5. Combining (5.10), (5.15) and (5.16), we get

$$
\begin{aligned}
& \chi_{\epsilon^{-1} Y^{\prime}}(\xi) \frac{B_{m}^{\epsilon} \mathbf{h}^{\epsilon}(\xi)}{\sqrt{1+\lambda_{m}^{\epsilon}(\xi)}} \rightarrow \frac{-i|Y|^{1 / 2}}{\sqrt{1+\frac{(\xi \cdot \hat{\eta})^{2}}{2|\hat{\eta}|^{4}} \lambda_{\hat{\eta}, m}^{\prime \prime}(0)}}\left\{\xi_{q} \mathcal{M}_{Y}\left(\mathfrak{a}_{p q r s}\right) \overline{\left(\boldsymbol{\Phi}_{\hat{\eta}, m}\right)_{p}}(y ; 0)\right. \\
&\left.+\xi_{l} \mathcal{M}_{Y}\left(\mathfrak{a}_{\text {pqrs }}(y) \frac{\partial}{\partial y_{q}}\left(\mathbf{w}^{k l}\right)_{p}(y)\right) \overline{\left(\boldsymbol{\Phi}_{\hat{\eta}, m}\right)_{k}}(0)\right\}\left(\frac{\partial \psi_{0}}{\partial x_{s}}(x) u_{r}^{*}(x)\right) .
\end{aligned}
$$

We recall the classical expressions for the homogenized coefficients $\mathfrak{b}_{\text {pqrs }}$ given in (3.1) here:

$$
\mathfrak{b}_{\text {pqrs }}=\mathcal{M}_{Y}\left(\mathfrak{a}_{p q r s}\right)+\mathcal{M}_{Y}\left(\mathfrak{a}_{p q l m}(y) \mathfrak{e}_{l m}\left(\mathbf{w}^{r s}(y)\right)\right)
$$

Thus we have the following weak convergence in $L^{2}\left(\mathbb{R}_{\xi}^{3}\right)$ :

$$
\lim _{\epsilon \rightarrow 0} \chi_{\epsilon^{-1} Y^{\prime}}(\xi) \frac{B_{m}^{\epsilon} \mathbf{h}^{\epsilon}(\xi)}{\sqrt{1+\lambda_{m}^{\epsilon}(\xi)}}=-\frac{-i|Y|^{1 / 2} \overline{\left(\mathbf{\Phi}_{\hat{\eta}, m}\right)_{p}}(0)}{\sqrt{1+\frac{(\xi \cdot \hat{\eta})^{2}}{2|\hat{\eta}|^{4}} \lambda_{\hat{\eta}, m}^{\prime \prime}(0)}} \xi_{q} \mathfrak{b}_{p q r s}\left(\frac{\partial \psi_{0}}{\partial x_{s}}(x) u_{r}^{*}(x)\right)
$$

Thus Lemma 5.2 is proved.

\subsection{Proof of Theorem $\mathbf{5 . 1}$}

We put together the results of the previous paragraph to find limits of the relations $(5.6)$, for $m=1,2,3$.

Step 1. Using the definition of $\lambda_{m}^{\epsilon}(\xi)$ in the equation (5.6) and dividing both sides by $\sqrt{1+\lambda_{m}^{\epsilon}(\xi)}$, for $m=1,2,3$, we have

$$
\chi_{\epsilon^{-1} Y^{\prime}}(\xi) \lambda_{m}^{\epsilon}(\xi) \frac{B_{m}^{\epsilon}\left(\psi_{0} \mathbf{u}^{\epsilon}\right)(\xi)}{\sqrt{1+\lambda_{m}^{\epsilon}(\xi)}}=\chi_{\epsilon^{-1} Y^{\prime}}(\xi) \frac{B_{m}^{\epsilon}\left(\psi_{0} \mathbf{f}\right)(\xi)+B_{m}^{\epsilon} \mathbf{g}^{\epsilon}(\xi)+B_{m}^{\epsilon} \mathbf{h}^{\epsilon}(\xi)}{\sqrt{1+\lambda_{m}^{\epsilon}(\xi)}}
$$

where we recall that

$$
\lambda_{m}^{\epsilon}(\xi)=\epsilon^{-2} \lambda_{\hat{\eta}, m}\left(\epsilon \frac{(\xi \cdot \hat{\eta})}{|\hat{\eta}|^{2}}\right) .
$$

We note that $A^{\epsilon}\left(\psi_{0} \mathbf{u}^{\epsilon}\right)$ is bounded in $\mathbf{H}^{-1}\left(\mathbb{R}^{3}\right)$ (refer Eq. (5.3)). Thanks to Lemma 2.7, it follows that right hand side of (5.18) is bounded in $L^{2}\left(\mathbb{R}^{3}\right)$. Therefore for a subsequence both sides of the relation (5.18) converge weakly in $L^{2}\left(\mathbb{R}_{\xi}^{3}\right)$.

As a corollary to the proof of Lemma 4.1, the left hand side of (5.18) converges pointwise for a subsequence as the sequence $\psi_{0} \mathbf{u}^{\epsilon}$ converges strongly in $\mathbf{L}^{2}\left(\mathbb{R}^{3}\right)$. 
Step 2. Using the expression (5.3), (5.7), and Lemma 5.2, we see that the right hand side of the equation (5.18) converges weakly in $L_{l o c}^{2}\left(\mathbb{R}_{\xi}^{3}\right)$ to

$$
\frac{|Y|^{1 / 2}}{\sqrt{1+\frac{(\xi \cdot \hat{\eta})^{2}}{2|\hat{\eta}|^{4}} \lambda_{\hat{\eta}, m}^{\prime \prime}(0)}}\left[\widehat{\psi_{0} f_{p}}(\xi)-\left(\sigma_{p q}^{*}\left(\widehat{x) \frac{\partial \psi_{0}}{\partial x_{q}}}\right)(\xi)-i \xi_{q} \mathfrak{b}_{p q r s}\left(\frac{\partial \psi_{0}}{\partial x_{s}} \widehat{(x) u_{r}^{*}(x)}\right)(\xi)\right] \overline{\left(\Phi_{\hat{\eta}, m}\right)_{p}}(0),\right.
$$

and the left hand side of the equation (5.18) converges weakly in $L_{\text {loc }}^{2}\left(\mathbb{R}_{\xi}^{3}\right)$ to

$$
\frac{|Y|^{1 / 2}}{\sqrt{1+\frac{(\xi \cdot \hat{\eta})^{2}}{2|\hat{\eta}|^{4}} \lambda_{\hat{\eta}, m}^{\prime \prime}(0)}}\left[\frac{(\xi \cdot \hat{\eta})^{2}}{|\hat{\eta}|^{4}} \frac{1}{2} \lambda_{\hat{\eta}, m}^{\prime \prime}(0) \widehat{\psi_{0} \mathbf{u}^{*}}(\xi)\right] \cdot \overline{\mathbf{\Phi}_{\hat{\eta}, m}}(0)
$$

In view of the relations (3.5) of Lemma 3.1, the expression in (5.20) is equal to

$$
\frac{|Y|^{1 / 2}}{\sqrt{1+\frac{(\xi \cdot \hat{\eta})^{2}}{2|\hat{\eta}|^{4}} \lambda_{\hat{\eta}, m}^{\prime \prime}(0)}}\left[\mathfrak{b}_{p q r s} \xi_{q} \xi_{s} \widehat{\psi_{0} u_{r}^{*}}(\xi)\right]_{p} \cdot \overline{\left(\Phi_{\hat{\eta}, m}\right)_{p}}(0)
$$

Step 3. We recall that for each fixed $\hat{\eta}$, the set $\left\{\boldsymbol{\Phi}_{\hat{\eta}, m}(0): m=1,2,3\right\}$ forms an orthonormal basis for $\mathbf{L}_{\#}^{2}(Y)$, and hence forms an orthogonal basis for $\mathbb{C}^{3}$, as they are independent of $y$. Consequently, (5.19) and (5.21) give, for each $p=1,2,3$,

$$
\mathfrak{b}_{p q r s} \xi_{q} \xi_{s} \widehat{\psi_{0} u_{r}^{*}}(\xi)=\widehat{\psi_{0} f_{p}}(\xi)-\left(\sigma_{p q}^{*}\left(\widehat{x)} \widehat{\frac{\partial \psi_{0}}{\partial x_{q}}}\right)(\xi)-i \xi_{q} \mathfrak{b}_{p q r s}\left(\frac{\partial \psi_{0}}{\partial x_{s}} \widehat{(x) u_{r}^{*}}(x)\right)(\xi) .\right.
$$

Step 4. By taking inverse Fourier transform in (5.22), we get

$$
\left(A^{*}\left(\psi_{0} \mathbf{u}^{*}\right)(x)\right)_{p}=\psi_{0} f_{p}(x)-\sigma_{p q}^{*}(x) \frac{\partial \psi_{0}}{\partial x_{q}}(x)-\mathfrak{b}_{p q r s} \frac{\partial}{\partial x_{q}}\left(\frac{\partial \psi_{0}}{\partial x_{s}}(x) u_{r}^{*}(x)\right),
$$

where the operator $A^{*}$ is defined by (5.2). By calculating via Leibnitz rule, we get

$$
\left(A^{*}\left(\psi_{0} \mathbf{u}^{*}\right)(x)\right)_{p}=\left(\psi_{0}(x) A^{*} u^{*}(x)\right)_{p}-\mathfrak{b}_{p q r s} \frac{\partial}{\partial x_{q}}\left(\frac{\partial \psi_{0}}{\partial x_{s}}(x) u_{r}^{*}(x)\right)-\mathfrak{b}_{p q r s} \frac{\partial \psi_{0}}{\partial x_{q}}(x) \frac{\partial u_{r}^{*}}{\partial x_{s}}(x) .
$$

From (5.23), (5.24), we have

$$
\psi_{0}(x)\left(A^{*} \mathbf{u}^{*}-\mathbf{f}\right)_{p}(x)=\left[\mathfrak{b}_{p q r s} \frac{\partial u_{r}^{*}}{\partial x_{s}}(x)-\sigma_{p q}^{*}(x)\right] \frac{\partial \psi_{0}}{\partial x_{q}}(x), p=1,2,3 .
$$

Step 5. Note that $\psi_{0} \mathrm{e}^{i x \cdot \omega} \in \mathcal{D}(\Omega)$ where $\omega$ is a unit vector in $\mathbb{R}^{3}$, and hence substituting in the relation (5.25), we get

$$
i \omega_{q} \psi_{0}(x)\left[\mathfrak{b}_{p q r s} \frac{\partial u_{r}^{*}}{\partial x_{s}}(x)-\sigma_{p q}^{*}(x)\right]=0, p=1,2,3
$$


Choosing $\omega$ from the standard ordered basis for $\mathbb{R}^{3}$, we get $\forall \psi_{0} \in \mathcal{D}(\Omega)$,

$$
\psi_{0}(x)\left[\mathfrak{b}_{p q r s} \frac{\partial u_{r}^{*}}{\partial x_{s}}(x)-\sigma_{p q}^{*}(x)\right]=0, p, q=1,2,3
$$

Let $x_{0} \in \Omega$ be arbitrary and let $\psi_{x_{0}} \in \mathcal{D}(\Omega)$ be such that $\psi_{x_{0}}$ is equal to one near $x_{0}$ and is zero outside a small neighbourhood of $x_{0}$. For $x$ belonging to a small neighbourhood of $x_{0}$, from the relation (5.26), we get

$$
\left[\mathfrak{b}_{p q r s} \frac{\partial u_{r}^{*}}{\partial x_{s}}(x)-\sigma_{p q}^{*}(x)\right]=0, p, q=1,2,3
$$

Since $x_{0} \in \Omega$ is arbitrary, we get

$$
A^{*} \mathbf{u}^{*}=\mathbf{f}, \text { and } \sigma_{p q}^{*}(x)=\mathfrak{b}_{p q r s} \frac{\partial u_{r}^{*}}{\partial x_{s}}(x), \quad \forall p, q=1,2,3 .
$$

Since the problem (5.2) has unique solution, and the homogenized coefficients $\mathfrak{b}_{\text {pqrs }}$ are independent of the subsequence used, it follows that the entire sequence $\mathbf{u}^{\epsilon}$ converges to $\mathbf{u}^{*}$.

Step 6. The symmetries of the homogenized coefficients follow from the alternate expressions for them given below.

$$
\mathfrak{b}_{p q r s}=\frac{1}{|Y|} \int_{Y} \mathfrak{a}_{u v l m}(y) \mathfrak{e}_{l m}\left(\mathbf{w}^{r s}(y)+\mathbf{y}^{r s}\right) \mathfrak{e}_{u v}\left(\mathbf{w}^{p q}(y)+\mathbf{y}^{p q}\right) \mathrm{d} y
$$

where $\mathbf{y}^{r s} \stackrel{\text { def }}{=} y_{r} \mathbf{e}_{s}+y_{s} \mathbf{e}_{r}$, and $\mathbf{e}_{r}, \mathbf{e}_{s}$ are the $r$ th and $s$ th basis vectors in the standard ordered basis for $\mathbb{R}^{3}$. This finishes the proof of the homogenization result Theorem 5.1.

\section{Higher modes do NOT CONTRIBUte}

As we have seen in the last section, the part of $\mathbf{u}^{\epsilon}$ which contributes to the homogenization process, denoted by $\mathbf{v}^{\epsilon}$, is its projection onto the space spanned by the Bloch modes indexed by 1,2 and 3 . It is interesting to know what happens to the rest of $\mathbf{u}^{\epsilon}$. This remainder part contains the components of $\mathbf{u}^{\epsilon}$ along higher modes present (i.e., modes with indices greater than or equal to 4 ). We denote the remainder by $\mathbf{E}^{\epsilon}(x)$ which is equal to $\mathbf{u}^{\epsilon}(x)-\mathbf{v}^{\epsilon}(x)$. That is,

$$
\mathbf{E}^{\epsilon}(x)=\sum_{m=4}^{\infty} \int_{\epsilon^{-1} Y^{\prime}} \mathfrak{B}_{m}^{\epsilon} \mathbf{u}^{\epsilon}(\xi) \mathrm{e}^{i x \cdot \xi} \boldsymbol{\Psi}_{m}^{\epsilon}(x ; \xi) \mathrm{d} \xi .
$$

The following result says that the $\mathbf{L}^{2}$-energy contained in these higher modes tends to zero in the homogenization limit.

Theorem 6.1. $\left\|\mathbf{E}^{\epsilon}\right\|_{\mathbf{L}^{2}\left(\mathbb{R}^{3}\right)} \leq C \epsilon$.

Referring to earlier works, $[8,25]$, we know that the existence of spectral gap between the lower part of the spectrum and the rest plays an important role in proving Theorem 6.1. In the case of the scalar equation treated 
in [8], eigenvalues with the usual ordering satisfied this requirement. In the case of systems, as will be seen below, Rellich branches possess the aforesaid spectral gap. More precisely we have:

Lemma 6.2. Let $\mathbf{u} \in \mathbf{H}_{\#}^{1}(\eta, Y)$ and $\mathbf{v} \in \mathbf{H}_{\#}^{1}(Y)$ be such that $\mathbf{u}(y)=\mathrm{e}^{i y \cdot \eta} \mathbf{v}(y)$. Then there exists a positive constant $C$ (independent of $\mathbf{u}, \mathbf{v}$ and $\eta \in Y^{\prime}$ ) satisfying

$$
C\left\{\|\nabla \mathbf{v}\|_{\mathbf{L}^{2}(Y)}+|\eta|\|\mathbf{v}\|_{\mathbf{L}^{2}(Y)}\right\} \leq\left\|\mathfrak{e}_{k l}(\mathbf{u})\right\|_{\mathbf{L}^{2}(Y)} \leq\left\{\|\nabla \mathbf{v}\|_{\mathbf{L}^{2}(Y)}+|\eta|\|\mathbf{v}\|_{\mathbf{L}^{2}(Y)}\right\} .
$$

Proof. Recalling the notation $\mathbf{u}=\left(u_{1}, u_{2}, u_{3}\right)$ and $\mathfrak{e}_{k l}(\mathbf{u})$ (see, (1.2)) from Section 1, we have

$$
\int_{Y} \mathfrak{e}_{k l}(\mathbf{u}) e_{k l}(\overline{\mathbf{u}}) \mathrm{d} y=\frac{1}{2} \int_{Y} \frac{\partial u_{k}}{\partial y_{l}} \frac{\overline{\partial u_{k}}}{\partial y_{l}} \mathrm{~d} y+\frac{1}{2} \int_{Y} \frac{\partial u_{k}}{\partial y_{l}} \overline{\frac{\partial u_{l}}{\partial y_{k}}} \mathrm{~d} y .
$$

Step 1. For each fixed $k=1,2,3$, we have the following inequality (see [7] p. 190):

$$
C\left\{\left\|\nabla v_{k}\right\|_{\mathbf{L}^{2}(Y)}+|\eta|\left\|v_{k}\right\|_{\mathbf{L}^{2}(Y)}\right\} \leq\left\|\nabla v_{k}+i \eta v_{k}\right\|_{\mathbf{L}^{2}(Y)}=\left\|\nabla u_{k}\right\|_{\mathbf{L}^{2}(Y)} .
$$

Adding the above inequalities for $k=1,2,3$, we get

$$
C\left\{\|\nabla \mathbf{v}\|_{\mathbf{L}^{2}(Y)}+|\eta|\|\mathbf{v}\|_{\mathbf{L}^{2}(Y)}\right\} \leq\|\nabla \mathbf{v}+i \eta \mathbf{v}\|_{\mathbf{L}^{2}(Y)}=\|\nabla \mathbf{u}\|_{\mathbf{L}^{2}(Y)}
$$

for some positive constant $C$. Thus, the first term in the RHS of (6.2) is bounded below.

Step 2. We now show that the second term in the RHS of (6.2) is non-negative by establishing

$$
\int_{Y} \frac{\partial u_{k}}{\partial y_{l}} \overline{\frac{\partial u_{l}}{\partial y_{k}}} \mathrm{~d} y=\int_{Y} \operatorname{div} \mathbf{u} \overline{\operatorname{div} \mathbf{u}} \mathrm{d} y \geq 0 .
$$

It is enough to prove (6.5) for smooth functions $\mathbf{u}$ as they are dense in $\mathbf{H}_{\#}^{1}(\eta, Y)$. Since $u_{k}=\mathrm{e}^{i y \cdot \eta} v_{k}$ by our notation, we have

$$
\begin{aligned}
\int_{Y} \frac{\partial u_{k}}{\partial y_{l}} \frac{\partial u_{l}}{\partial y_{k}} \mathrm{~d} y & =\int_{Y}\left(\frac{\partial v_{k}}{\partial y_{l}}+i \eta_{l} v_{k}\right) \overline{\left(\frac{\partial v_{l}}{\partial y_{k}}+i \eta_{k} v_{l}\right)} \mathrm{d} y \\
& =\int_{Y} \frac{\partial v_{k}}{\partial y_{l}} \frac{\partial v_{l}}{\partial y_{k}} \mathrm{~d} y+i \eta_{l} \int_{Y} v_{k} \frac{\partial \bar{v}_{l}}{\partial y_{k}} \mathrm{~d} y-i \eta_{k} \int_{Y} \bar{v}_{l} \frac{\partial v_{k}}{\partial y_{l}} \mathrm{~d} y+\eta_{k} \eta_{l} \int_{Y} v_{k} \bar{v}_{l} \mathrm{~d} y .
\end{aligned}
$$

We now perform integration by parts twice in the first term, once in second and third terms in (6.6). In this process there will be no boundary contributions as the integrands are periodic functions. As a result, we get

$$
\begin{aligned}
\int_{Y} \frac{\partial u_{k}}{\partial y_{l}} \frac{\overline{\partial u_{l}}}{\partial y_{k}} \mathrm{~d} y & =\int_{Y} \frac{\partial v_{k}}{\partial y_{k}} \frac{\overline{\partial v_{l}}}{\partial y_{l}} \mathrm{~d} y-i \eta_{l} \int_{Y} \bar{v}_{l} \frac{\partial v_{k}}{\partial y_{k}} \mathrm{~d} y+i \eta_{k} \int_{Y} v_{k} \frac{\partial \bar{v}_{l}}{\partial y_{l}} \mathrm{~d} y+\eta_{k} \eta_{l} \int_{Y} v_{k} \bar{v}_{l} \mathrm{~d} y \\
& =\int_{Y}\left(\frac{\partial v_{k}}{\partial y_{k}}+i \eta_{k} v_{k}\right) \overline{\left(\frac{\partial v_{l}}{\partial y_{l}}+i \eta_{l} v_{l}\right)} \mathrm{d} y \\
& =\int_{Y} \operatorname{div} \mathbf{u} \overline{\operatorname{div} \mathbf{u}} \mathrm{d} y .
\end{aligned}
$$


In view of the relations (6.4) and (6.5), the first inequality in (6.1) follows. The second inequality in (6.1) is obvious.

Lemma 6.3. Let $\lambda_{m}($.$) for m \in \mathbb{N}$ be as introduced in the Section 2. Then there exists a constant $\nu>0$ such that

$$
\lambda_{m}(\eta) \geq \nu, \quad \forall m \geq 4 \text { and } \forall \eta \in Y^{\prime}
$$

Proof.

Step 1. Thanks to Lemma 6.2 and uniform ellipticity of the elastic tensor, we get

$$
a(\eta)(\mathbf{v}, \mathbf{v}) \geq C\left\{\|\nabla \mathbf{v}\|_{\mathbf{L}^{2}(Y)}^{2}+|\eta|^{2}\|\mathbf{v}\|_{\mathbf{L}^{2}(Y)}^{2}\right\}
$$

In view of the relation $\lambda_{m}(\eta)=a(\eta)\left(\mathbf{\Phi}_{\mathbf{m}}(. ; \eta), \mathbf{\Phi}_{\mathbf{m}}(. ; \eta)\right)$, the inequality (6.8) yields

$$
\lambda_{m}(\eta) \geq C|\eta|^{2}, \quad \forall m \in \mathbb{N} \text { and } \forall \eta \in Y^{\prime} .
$$

Step 2. Let $\mu_{m}(\eta)$ for $m \in \mathbb{N}$ be the ordered spectrum of the operator $A(\eta)$ (see, Lem. 1.1). Since each of $\mu_{m}(\eta)$ is continuous in $\eta$ and since $0=\mu_{1}(0)=\mu_{2}(0)=\mu_{3}(0)<\mu_{4}(0)$, there exist a $\mu^{*} \in \mathbb{R}$ and a ball $B$ with centre at $\eta=0$ and radius $r$ for some $r>0$ such that

$$
\mu_{1}(\eta) \leq \mu_{2}(\eta) \leq \mu_{3}(\eta)<\mu^{*}<\mu_{4}(\eta) \leq \mu_{5}(\eta) \leq \ldots \quad \forall \eta \in B
$$

In particular, the inequalities (6.10) say that $\mu^{*}$ is not an eigenvalue of $A(\eta)$ and there are only three eigenvalues counting multiplicities which are less than $\mu^{*}$ for $\eta \in B$. At $\eta=0$, we have the following equalities (see (1.15)):

$$
\lambda_{1}(0)=\lambda_{2}(0)=\lambda_{3}(0)=0=\mu_{1}(0)=\mu_{2}(0)=\mu_{3}(0) .
$$

Recall that Rellich branches $\left\{\lambda_{m}(\eta)\right\}_{m \in \mathbb{N}}$ also give a listing of all the eigenvalues of the operator $A(\eta)$ including multiplicities and hence is a permutation of $\left\{\mu_{m}(\eta)\right\}_{m \in \mathbb{N}}$ for all $\eta \in Y^{\prime}$. If we restrict $\eta$ to $B$, then we claim that $\lambda_{1}(\eta), \lambda_{2}(\eta), \lambda_{3}(\eta)$ is a permutation of $\mu_{1}(\eta), \mu_{2}(\eta), \mu_{3}(\eta)$. If this were not the case then for some $m=1,2,3$ and $\eta_{0} \in B, \lambda_{m}\left(\eta_{0}\right)>\mu^{*}$. Letting $\eta_{0}=\rho_{0} \hat{\eta}_{0}$, by definition of $\lambda_{m}($.$) , we have \lambda_{\hat{\eta}_{0}, m}\left(\rho_{0}\right)>\mu^{*}$. Since the map $\rho \longmapsto \lambda_{\hat{\eta}_{0}, m}(\rho)$ is continuous, there exists a $\rho_{1}<\rho_{0}$ (as a consequence, $\rho_{1} \hat{\eta}_{0} \in B$ ) such that $\lambda_{\hat{\eta}_{0}, m}\left(\rho_{1}\right)=\mu^{*}$. This contradicts the fact that $\mu^{*}$ is not an eigenvalue for $\eta \in B$, and hence our claim follows. As a consequence,

$$
\lambda_{m}(\eta) \geq \mu^{*}, \quad \forall m \geq 4 \text { and } \forall \eta \in B
$$

The relation (6.11) provides a lower bound for $\lambda_{m}(\eta)(m \geq 4)$ for $\eta \in B$ and (6.9) gives rise to a lower bound for $\eta$ outside $B$. Thus we obtain (6.7).

Proof of Theorem 6.1. Thanks to the spectral gap asserted by Lemma 6.3, we obtain the required estimate as follows. We have the relation

$$
\int_{\mathbb{R}^{3}}\left(A^{\epsilon} \mathbf{u}^{\epsilon}\right)_{p}(x) \mathbf{u}_{p}^{\epsilon}(x) \mathrm{d} x=\int_{\mathbb{R}^{3}} f_{p}(x) \mathbf{u}_{p}^{\epsilon}(x) \mathrm{d} x .
$$

Since the elasticity tensor is bounded and the sequence $\mathbf{u}^{\epsilon}$ is bounded in the space $\mathbf{H}^{1}\left(\mathbb{R}^{3}\right)$, we get

$$
\int_{\mathbb{R}^{3}} f_{p}(x) \mathbf{u}_{p}^{\epsilon}(x) \mathrm{d} x \leq c\left\|\mathbf{u}^{\epsilon}\right\|_{\mathbf{H}^{1}\left(\mathbb{R}^{3}\right)} \leq C .
$$


Taking the $m$ th Bloch transform on the equation $A^{\epsilon} \mathbf{u}^{\epsilon}(x)=f(x)$ in $\mathbb{R}^{3}$ yields

$$
\lambda_{m}^{\epsilon}(\xi) \mathfrak{B}_{m}^{\epsilon} \mathbf{u}^{\epsilon}(\xi)=\mathfrak{B}_{m}^{\epsilon} \mathbf{f}(\xi) .
$$

Now, by Plancheral identity (2.4), the estimate (6.12) takes the form

$$
\int_{\epsilon^{-1} Y^{\prime}} \sum_{m=1}^{\infty} \mathfrak{B}_{m}^{\epsilon} \mathbf{f}(\xi) \overline{\mathfrak{B}_{m}^{\epsilon} \mathbf{u}^{\epsilon}(\xi)} \mathrm{d} \xi \leq C .
$$

Since $\lambda_{m}^{\epsilon}(\xi) \geq 0$ for $m \in \mathbb{N}$, we have

$$
\int_{\epsilon^{-1} Y^{\prime}} \sum_{m=1}^{\infty} \mathfrak{B}_{m}^{\epsilon} \mathbf{f}(\xi) \overline{\mathfrak{B}_{m}^{\epsilon} \mathbf{u}^{\epsilon}(\xi)} \mathrm{d} \xi=\int_{\epsilon^{-1} Y^{\prime}} \sum_{m=1}^{\infty} \lambda_{m}^{\epsilon}(\xi)\left|\mathfrak{B}_{m}^{\epsilon} \mathbf{u}^{\epsilon}(\xi)\right|^{2} \mathrm{~d} \xi \geq \int_{\epsilon^{-1} Y^{\prime}} \sum_{m=4}^{\infty} \lambda_{m}^{\epsilon}(\xi)\left|\mathfrak{B}_{m}^{\epsilon} \mathbf{u}^{\epsilon}(\xi)\right|^{2} \mathrm{~d} \xi .
$$

Thanks to the inequality (6.7), in view of the relation $\lambda_{m}^{\epsilon}(\xi)=\epsilon^{-2} \lambda_{m}(\epsilon \xi)$, we have

$$
\int_{\epsilon^{-1} Y^{\prime}} \sum_{m=4}^{\infty} \lambda_{m}^{\epsilon}(\xi)\left|\mathfrak{B}_{m}^{\epsilon} \mathbf{u}^{\epsilon}(\xi)\right|^{2} \mathrm{~d} \xi \geq \epsilon^{-2} \nu \int_{\epsilon^{-1} Y^{\prime}} \sum_{m=4}^{\infty}\left|\mathfrak{B}_{m}^{\epsilon} \mathbf{u}^{\epsilon}(\xi)\right|^{2} \mathrm{~d} \xi=\epsilon^{-2} \nu\left\|\mathbf{E}^{\epsilon}\right\|_{\mathbf{L}^{2}\left(\mathbb{R}^{3}\right)} .
$$

Now Theorem 6.1 follows from the above inequality and (6.13).

Acknowledgements. The authors acknowledge with thanks the discussions with Professor G. Allaire, École Polytechnique, Paris. The authors wish to thank the anonymous referee for suggestions which improved the overall presentation of the paper.

\section{REFERENCES}

[1] G. Allaire, Homogenization and two scale convergence. SIAM J. Math. Anal. 23 (1992) 1482-1518.

[2] G. Allaire and C. Conca, Bloch wave homogenization for a spectral problem in fluid-solid structures. Arch. Rational Mech. Anal. 135 (1996) 197-257.

[3] G. Allaire and C. Conca, Boundary layers in the homogenization of a spectral problem in fluid-solid structures. SIAM J. Math. Anal. 29 (1997) 343-379.

[4] A. Bensoussan, J.L. Lions and G. Papanicolaou, Asymptotic analysis for periodic structures. North Holland, Amsterdam (1978).

[5] C. Conca, S. Natesan and M. Vanninathan, Numerical solution of elliptic partial differential equations by Bloch waves method, XVII CEDYA: Congress on differential equations and applications/VII CMA: Congress on applied mathematics, Dep. Mat. Appl., Univ. Salamanca, Salamanca (2001) 63-83.

[6] C. Conca, R. Orive and M. Vanninathan, Bloch approximation in homogenization and applications. SIAM J. Math. Anal. 33 (2002) 1166-1198.

[7] C. Conca, J. Planchard and M. Vanninathan, Fluids and periodic structures. John Wiley \& Sons, New York, and Masson, Paris (1995).

[8] C. Conca and M. Vanninathan, Homogenization of periodic structures via Bloch decomposition. SIAM J. Appl. Math. 57 (1997) 1639-1659.

[9] C. Conca and M. Vanninathan, Fourier approach to homogenization. ESAIM: COCV 8 (2002) 489-511.

[10] A.P. Cracknell and K.C. Wong, The Fermi surface. Clarendon press, Oxford (1973).

[11] G. Dal maso, An introduction to $\Gamma$-convergence. Birkhäuser, Boston (1993).

[12] P. Gérard, Microlocal defect measures. Commun. PDE 16 (1991) 1761-1794.

[13] P. Gérard, P.A. Markowich, N.J. Mauser and F. Poupaud, Homogenization limits and Wigner transforms. Comm. Pure Appl. Math. 50 (1997) 323-379.

[14] V.V. Jikov, S.M. Kozlov and O.A. Oleinik, Homogenization of Differential operators and Integral functionals. Berlin, Springer-Verlag (1994).

[15] T. Kato, Perturbation theory for linear operators. 2nd edition, Springer-Verlag, Berlin (1980). 
[16] F. Murat and L. Tartar, H-Convergence, Topics in the Mathematical Modeling of Composite Materials, A. Charkaev and R. Kohn Eds. PNLDE 31, Birkhäuser, Boston (1997).

[17] G. Nguetseng, A general convergence result for a functional related to the theory of homogenization. SIAM J. Math. Anal. 20 (1989) 608-623.

[18] O.A. Oleinik, A.S. Shamaev and G.A. Yosifian, Mathematical problems in elasticity and homogenization. North Holland, Amsterdam (1992).

[19] F. Rellich, Perturbation theory of eigenvalue problems. Gordon and Breach science publishers, New York (1969).

[20] M. Roseau, Vibrations in Mechanical systems: Analytical methods and applications. Springer-Verlag, Berlin (1987).

[21] W. Rudin, Functional analysis. 2nd edition, Mc-Graw Hill, New York (1991).

[22] J. Sánchez-Hubert and E. Sánchez-Palencia, Vibration and coupling of continuous systems: asymptotic methods. Springer-Verlag, Berlin (1989).

[23] E. Sánchez-Palencia, Non-homogeneous media and vibration theory. Lect. Notes Phys. 127 (1980).

[24] F. Santosa and W.W. Symes, A dispersive effective medium for wave propagation in periodic composites. SIAM J. Appl. Math. 51 (1991) 984-1005.

[25] S. Sivaji Ganesh and M. Vanninathan, Bloch wave homogenization of scalar elliptic operators. Asymptotic Analysis 39 (2004) $15-44$.

[26] L. Tartar, H-measures, a new approach for studying homogenization, oscillations and concentration effects in partial differential equations. Proc. Roy. Soc. Edin. Sect. A 115 (1990) 193-230.

[27] N. Turbé, Applications of Bloch decomposition to periodic elastic and viscoelastic media. Math. Meth. Appl. Sci. 4 (1982) 433-449. 